\title{
Presseförderung als Mittel gegen Medienkonzentration und für die publizistische Vielfalt? Quasi-experimentelle Untersuchung der Schweiz im Vergleich zu Belgien, Österreich, Irland sowie Deutschland
}

\author{
Steffen Kolb, Hochschule für Technik und Wirtschaft (HTW) Berlin, FB Informatik, Kommunikation \\ und Wirtschaft | Wirtschaftskommunikation* \\ Mathias Oertel, Hochschule für Technik und Wirtschaft (HTW) Berlin, FB Informatik, Kommunikation \\ und Wirtschaft | Wirtschaftskommunikation
}

*korrespondierender Autor: Steffen.Kolb@HTW-Berlin.de

\begin{abstract}
Mass media play a certain role for society in democratic systems. Communication research faces this role by analyzing media performance. Well performing media provide the society with a diversity of topics, opinions, and sources. Mostly economically driven processes of media concentration endanger media diversity by decreasing the amount of independent media companies most probably causing less diverse media content. To counteract processes of media concentration in the field of daily newspapers many countries have established various kinds of press subsidies. This study examines empirically what kind of impact press subsidies have on media concentration. It uses a quasi-experimental design in a comparative approach including data from Switzerland, Belgium, Austria, Ireland, and Germany.
\end{abstract}

Keywords

media concentration, pluralism, press subsidies, media performance, state aid, daily press

\section{Einleitung und Problemstellung}

Im Jahr 2009 verkündete die in der Schweiz bereits marktführende Tamedia AG den Zukauf der Edipresse Suisse. Mit diesem Übernahmeprozess, welcher am 1. Januar 2012 abgeschlossen wurde, zementierte die Tamedia AG ihre Marktführung auch im Bereich Tageszeitungen über die Sprachgrenzen hinweg (vgl. Tamedia AG, 2014). In Deutschland übernahm zum 1. Januar 2014 die Funke Mediengruppe ${ }^{1}$ zwei Tageszeitungen (Berliner Morgenpost, Hamburger Abendblatt) sowie fünf weitere Zeitschriften der Axel Springer AG (vgl. Axel Springer AG, 2013). Für 2014 konstatiert Röper zum zweiten Mal in Folge die stärkste Medienkonzentration in Deutschland (vgl. Röper, 2014, 2012). In Frankreich deutete sich 2014 die Über-

1 Die Funke Mediengruppe firmierte bis einschliesslich des Jahres 2012 unter dem Namen WAZ-Gruppe. Aus diesem Grund wird in den Ergebnissen bis zum Jahr 2012 der alte Name verwendet. nahme der reichweitenstärksten Gratistageszeitung 20minutes durch die Groupe Bolloré und TF1 an (vgl. Debouté \& Renault, 2014), schliesslich verkaufte Schibsted seine Anteile an den grössten frankophonen Medienkonzern in Belgien, die Rossel-Gruppe (vgl. Groupe Rossel, 2015). Das sind nur einige Beispiele, die einen international wahrnehmbaren Trend der Medienkonzentration im Printbereich widerspiegeln.

Aus ökonomischer Perspektive ermöglicht die Ballung von Ressourcen und Kräften Synergien, welche zu grösserer wirtschaftlicher Effizienz führen (vgl. z. B. Altmeppen, 2013, S. 211-213; Frühschütz, 2004, S. 242; Puppis, 2010, S. 86). Insbesondere in Zeiten starken Drucks auf die Branche handelt es sich bei Zusammenschlüssen, Aufkäufen oder Joint Ventures also um ökonomisch rationale Entscheidungen (vgl. Murschetz, 2013a, S. 3-8). Medienkonzentration gibt es aber auch bei den audiovisuellen Medien bzw. als Verflechtung durch den gesamten Medien- 
bereich, wie das Schweizer Beispiel der Aufkäufe von TeleBärn und TeleZüri durch die in Print, Radio, TV und Online aktiven AZ-Medien belegt (vgl. Kust \& Kolb, 2013, S. 24).

Aus demokratietheoretischer und damit auch gesellschaftlicher Perspektive erwächst allerdings ein nachhaltiges Problem aus diesem Trend: Medienkonzentrationsprozesse führen zu einer Verringerung der Anbieter am Markt. Damit stehen solche Prozesse einer publizistischen (Meinungs-)Vielfalt und somit der Erfüllung gesellschaftlich relevanter Funktionen der Medien für Demokratien eher entgegen. Zumindest entsteht ein Spannungsverhältnis zwischen beiden (vgl. Russi, 2013, S. 13-14). Im Pressesektor wird fast in ganz Europa medienpolitisch versucht, solchen Konzentrationsprozessen durch spezifische Fördermassnahmen ${ }^{2}$ entgegenzuwirken, um auf diesem Wege publizistische Vielfalt zu fördern oder zumindest zu erhalten (vgl. z.B. Murschetz, 1998; Puppis, 2010; WAN-IFRA, 2015). Auch ausserhalb von Europa gibt es Presseförderung, wie der Überblick von Künzler und Kollegen (2013) oder Murschetz (vgl. 2013c) belegen. In letzterem werden allerdings u.a. spieltheoretisch begründete Bedenken publiziert, ob Presseförderung überhaupt als ökonomisches Instrument die wünschenswerte Entwicklung fördern kann (vgl. Murschetz, 2013b).

Fest steht, dass es wahrnehmbare Medienkonzentrationsprozesse gibt und dass die Medienpolitik gezielt Presseförderungsmassnahmen einsetzt, um diesen entgegenzuwirken. Es erscheint daher sinnvoll, auch empirisch zu erforschen, inwiefern diese tatsächlich eine in der Realität messbare Wirkung entfachen. Das Ziel dieses Beitrages ist also, anhand statistischer Daten über die Zeit und über Ländergrenzen hinweg $\mathrm{zu}$ erforschen, welchen Einfluss Presseförderungsmass-

2 Auf die jeweiligen Arten der Presseförderung (indirekt, direkt; allgemein, selektiv) wird an dieser Stelle nicht vertiefend eingegangen. Für ein weiteres Verständnis sind diese z. B. den grundlegenden Artikeln von Murschetz (2013b) und Holtz-Bacha (1994) zu entnehmen. nahmen auf (einen Teil der $)^{3}$ Medienkonzentration und damit auf einen noch kleineren Ausschnitt publizistischer Vielfalt haben, wobei die Schweiz im Mittelpunkt der vergleichenden Untersuchung steht.

\section{Kommunikationswissenschaftlicher Hintergrund}

Massenmedien werden in der kommunikationswissenschaftlichen Auseinandersetzung mit ihren gesellschaftlichen Funktionen in modernen Demokratien unter anderem als zwingendes Mittel zum Austausch zwischen den politischen Institutionen und der Bevölkerung durch das Herstellen von Öffentlichkeit beschrieben (vgl. z. B. Beck, 2013, S. 98-99). Imhof, Blum, Bonfadelli und Jarren bringen das Verhältnis zwischen Öffentlichkeit und Demokratie auf den Punkt: «Ohne freie Öffentlichkeit [...] keine demokratische Gesellschaft» (2006, S. 12). Mit diesem Verständnis der medialen Funktionen geht einher, dass die Qualität von Medienleistungen dann als das (gute) Erfüllen von Funktionen bezeichnet werden kann (vgl. Schwarb, 2007, S. 81-88). Unter dem Begriff "Media Performance" (McQuail, 1992) hat sich hierzu eine ganze Forschungsrichtung ausgebildet (vgl. aktuell z.B. Puppis, Künzler, \& Jarren, 2013), auf die an dieser Stelle nicht weiter eingegangen werden

3 Klar ist, dass es sich beim hier untersuchten Tageszeitungsmarkt nur um einen Ausschnitt des Medienmarktes handelt. Insbesondere mit Blick auf die folgende theoretischen Rahmensetzung und auch die Mediennutzung müssten eigentlich alle Mediengattungen einbezogen werden. Zum jetzigen Kenntnisstand ist ein solch ambitioniertes Vorgehen in Kombination mit einem Längsschnitt- und einem Ländervergleich kaum durchführbar und auch nicht zielführend, da zu viele intervenierende Variablen in den Vergleichssettings betrachtet werden müssten. Es handelt sich also um eine vereinfachte Betrachtung, die aber durchaus als "state of the art" bezeichnet werden kann, wie der umfangreiche internationale Forschungsstand an Vielfaltsanalysen im Fernsehbereich zeigt (vgl. Kolb, 2015). 
muss. Die Medienperformanz bezieht grundsätzlich alle publizistischen Medien ein, so dass mit der hier angestrebten Betrachtung der Tagespresse nur ein Teil der Leistungen untersucht werden kann und z.B. das Fernsehen in seinen publizistischen Leistungen in verschiedenen europäischen Ländern nicht zu unterschätzen ist (vgl. Kolb, 2015).

Für eine funktionierende, repräsentative Demokratie macht die Kommunikationswissenschaft basierend, unter anderem, auf dem Pluralismus strukturell publizistische Vielfalt als einen oder gar den entscheidenden Leistungs- bzw. "Performance"-Aspekt aus (vgl. z. B. Rager \&Weber, 1992; Zerback, 2013): Eine garantierte Vielfalt an Meinungen, Perspektiven und Inhalten ist für «die umfassende Information der Bürger und für Meinungsbildungsprozesse in demokratisch organisierten Staaten unentbehrlich» (Bonfadelli \& Schwarb, 2006, S. 22). Eine solche publizistische Vielfalt lässt sich nach Zerback (2013, S. 9-10) in ein enges und ein weites Verständnis unterteilen: Das enge Verständnis stellt dabei ausschliesslich auf die inhaltliche Ebene ab - kurz: inhaltliche Vielfalt -, da letztlich diese primär den gesellschaftlichen Nutzen hinsichtlich der Funktion der Medien generiert. Das weite Verständnis schliesst darüber hinaus die medialen Strukturen mit ein - kurz: strukturelle Vielfalt (vgl. auch Kolb, 2015, S. 45-49).

Diese Kategorisierung findet sich ebenfalls in McQuails (1992, S. 144-145) Sicht aufVielfalt. Er definiert sie wie folgt:

1. als Zugang zu Medienangeboten für alle gesellschaftlich relevanten Gruppen (diversity as access) - was sich auf einen freien, allgemeinen und nicht-exklusiven Zugang $\mathrm{zu}$ Medienangeboten bezieht (vgl. hierzu z. B. auch Freedman, 2008, S. 7);

2. als vielfältige Auswahlmöglichkeiten für die Rezipienten (diversity as more channels and choice for the audience) was sich direkt auf die strukturelle Ebene publizistischer Vielfalt bezieht;

3. als die vielfältige Repräsentation der Gesellschaft in all ihren Facetten (" $d i$ - versity as reflection) - was sich nach Sarcinelli (1998) einesteils auf eine «Richtungspolitische Pluralität» bezieht, also auf die Repräsentation der Vielzahl an unterschiedlichen politischen Richtungstendenzen einer Gesellschaft, sowie anderenteils auf eine «Pluralität von Komplexitätsgraden» (S. 12), also die Abbildung aller Komplexitätsgrade im medialen Angebot um die verschiedenen in der Gesellschaft vorkommenden "Adressatengruppen und Teilöffentlichkeiten erreichen [zu] können» (S. 12).

Der erste Aspekt (diversity as access) kann bei der Untersuchung westlicher Demokratien ausser Acht gelassen werden, da hier von einem allgemeinen und unbeschränkten Zugang zu den Massenmedien ausgegangen werden kann. Hinsichtlich des dritten Aspekts (diversity as reflection) geht es um die Ebene der Medieninhalte oder das oben genannte enge Verständnis publizistischer Vielfalt -, die hier aus forschungsökonomischen Gründen ebenfalls ausgeklammert werden muss, um die Frage nach der publizistischen Vielfalt in die Breite analysierbar zu machen. ${ }^{4}$

Der zweite Aspekt (diversity as more channels and choice for the audience) spricht ganz allgemein die Angebotsebene an: Eine Vielfältigkeit der Auswahlmöglichkeiten beim Rezipienten dürfte - bei gewährleisteter Zugangsvielfalt - dann gegeben sein, wenn ein vielfältiges Angebot vorliegt (vgl. Kolb, 2015, S. 23). Die einfachste Herangehensweise an publizistische Vielfalt setzt voraus, dass durch mehr voneinander unabhängige Anbieter auf einem Markt oder eine grössere strukturelle Vielfalt quasi automatisch die inhaltliche Vielfalt gesteigert wird (vgl. z. B. Holtz-Bacha, 1994, S. 445; Knoche, 1999, S. 136-137; Napoli, 1999, S. 8; Napoli, 2001, S. 133). Diese Argumentation ist aussenpluralistisch ${ }^{5}$ und verleiht

4 Eine inhaltliche und methodische Begründung für die Beschränkung auf die Analyse der Meso- und Makroebene ist bereits publiziert (vgl. Kolb \& Oertel, 2016, S. 143-144).

5 Binnenpluralistische Herangehensweisen fokussieren die Vielfalt innerhalb eines $\mathrm{Me}$ - 
dem zweiten Aspekt McQuails (siehe oben) eine entscheidende Bedeutung im Hinblick auf diese Untersuchung: Auf der einen Seite lässt sich strukturelle Vielfalt (leichter) quantifizieren, auf der anderen Seite lässt sie - bei Zugrundelegung des eben beschriebenen Zusammenhangs Schlüsse auf eine inhaltliche Vielfalt zu. Das ist eine gängige Vorgehensweise auf der Meso- bzw. der Makroebene (vgl. Kolb, 2015, S. 49-51).

Auf dieser Basis erfolgt die normativ-analytische Fernsehforschung, welche im deutschsprachigen Raum hauptsächlich kontinuierlich im Auftrag der Aufsichtsbehörden durchgeführt wird (vgl. für die Schweiz ausführlich Trebbe et al., 2008). In der theoretischen Erweiterung wird in Bezug auf das Fernsehen zunächst die «originäre Sendeleistung» (Kolb, 2015, S. 130) betrachtet, die kurzfristige Wiederholungen des Programms innerhalb einer Woche aus der Analyse herausrechnet. Im zweiten Schritt werden die Zeitanteile der Fernsehpublizistik, also aller potenziell informierenden nicht fiktionalen Programmangebote genauer betrachtet (vgl. Kolb, 2015). Damit lassen sich erste Aussagen über die potenziellen Leistungen treffen: Ein Programm $A$ mit fünf Minuten Nachrichten pro Tag kann kaum mehr an gesellschaftlich relevanter Berichterstattung erbringen als Programm $B$ mit fünf Stunden. Sicher sein kann man sich aber nicht, weil die fünf Stunden Nachrichten am Tag bei Sender $B$ auch vollständig mit gesellschaftlich Irrelevantem bestritten werden könnten, die fünf Minuten von $A$ aber zumindest das Wichtigste in Kurzform bringen könnten. Daher geht die normativ-analytische Fernsehforschung nach den Strukturen bzw. den Genres und Formaten auf die Themen der einzelnen Beiträge in den Sendungen ein (vgl. Kolb, 2015, S. 209-270).

Übertragen auf die Tagespresse wird hier erstens davon ausgegangen, dass es keine Wiederholungen geben kann und zweitens, dass die gesamte Zeitung po-

dienprodukts. Sie sind damit der inhaltlichen Betrachtung zuzuordnen, die hier ausgeklammert werden muss. tenziell gesellschaftlich Relevantes beinhalten kann. Somit lassen sich also statistische Daten zu gesamten Tageszeitungen als Grundlage für Analysen rechtfertigen, auch wenn damit über die inhaltliche Leistungsfähigkeit der angebotenen Zeitungen keine Aussagen getroffen werden können. Je weniger Zeitungen es also auf dem Markt gibt, desto weniger vielfältig in struktureller Hinsicht ist das Printmedienangebot, wie auch Röper (vgl. 2014) dies für seine zeitvergleichenden Analysen operationalisiert.

Es gibt eine Reihe von Versuchen, sich der Analyse von interner und externer Vielfalt international vergleichend zu nähern. Allerdings haben solche Studien in der Regel (mindestens) zwei grundlegende Probleme, die hier kurz skizziert werden sollen. ${ }^{6}$ Das erste Problem ist in der Regel die Daten- und sogar die Definitionsbasis (vgl. Aslama et al., 2007), da es keine-oder allenfalls Vorschläge ${ }^{7}$ für - internationale(n) Standards zur Messung von Vielfalt gibt und damit die Vergleichbarkeit der Ergebnisse nicht gewährleistet werden kann. Daraus entsteht für die Konzeption international vergleichender Forschungsbeiträge oft ein zweites Problem: die Verwendung von Länderberichten mit oder ohne vergleichendem Fazit (vgl. z. B. Aslama et al., 2007). Die Resultate sind darum ernüchternd: Sowohl Wettbewerb als auch Medienkonzentration können $\mathrm{zu}$ mehr aber auch zu weniger Vielfalt führen (vgl. Aslama et al., 2007, S. 93). Vermutlich können Sie auch dazu führen, dass alles beim Alten bleibt, womit die Forschungsfrage für diesen Beitrag auf der Hand liegt: Welchen Effekt hat eigentlich ein Instrument,

6 Dies erfolgt auf Wunsch eines Reviewers, um zu verdeutlichen, warum der hier vorgeschlagene Weg und dieser Beitrag von grosser Relevanz für die Kommunikationswissenschaft sind.

7 Einen solchen Vorschlag machen van Cuilenburg und van der Wurff (vgl. 2007), wobei es sich mehr oder weniger um den Vorschlag handelt, das niederländische System auf alle anderen anzuwenden. Leider verwenden die Autoren dabei Messindizes, die von der Anzahl der Anbieter abhängig sind und daher ungeeignet (vgl. Kolb, 2015). 
das zur Vielfaltssicherung eingesetzt wird? Dazu ist es auch von Bedeutung, die propagierten Indexberechnungen (vgl. van Cuilenburg, 2007) und verschiedene Berechnungsarten zu erproben, um Fehlschlüssen vorzubeugen. Kolb (2015) hat dies in seiner vergleichenden Fernsehstudie von acht Ländern gezeigt. Es kann auch exemplarisch verdeutlicht werden: Das intern vielfältigste Fernsehvollprogramm in Deutschland auf Grundlage von indexbasierten Entropieberechnungen ist RTL II.

\section{Medienökonomischer Hintergrund}

Im vorliegenden Beitrag werden nationale Pressemärkte betrachtet. Ob das aus ökonomischer Perspektive immer die beste Einteilung ist, bezweifelt schon Röper (2014), wenn er auf die Arbeit von Schütz (2012) hinweist, die die Verbreitungsgebiete der Zeitungen als relevante Märkte identifiziert. Für die Schweiz wird dies ähnlich in Bezug auf das Fernsehen diskutiert, wenn Trebbe und Kollegen (vgl. 2008) von drei sprachlich getrennten Fernsehmärkten ausgehen und für die regionalen Anbieter Wettbewerbsanalysen für sich überschneidende Verbreitungsgebiete vorgenommen werden (vgl. Kolb \& Lüdi, 2016). Für Tageszeitungen wären analog in Detailanalysen also mindestens vier (sprachlich getrennte) oder noch deutlich mehr (durch Verbreitungsgebiete getrennte) Märkte zu betrachten. Für einen internationalen Vergleich eignet sich ein solcher Detailgrad nicht, hier funktionieren nur sehr grobe nationale Betrachtungen (vgl. auch Murschetz, 2013c), zumal in detaillierter Betrachtung durch viele «Einzeitungskreise» die Anbietervielfalt oftmals gleich Null wäre.

Eine ausführliche Beleuchtung des medienökonomischen Hintergrunds müsste eine dezidierte Unterscheidung zwischen verschiedenen ökonomischen Strömungen und deren Bewertungen von staatlichen Interventionen in den Markt vornehmen, da hier zum Teil mit stark unterschiedlichen Vorannahmen gearbeitet wird. Das lässt sich beispielhaft an der
Einschätzung Picards (vgl. 2006) zeigen, der zum Schluss kommt, dass öffentliche Presseförderung nicht oder wenig geeignet ist, den Printmedien zu helfen. Murschetz (2013b, S. 41) gibt in seiner Zusammenfassung als einen von Picards Gründen an, dass öffentliche Subventionierung generell schlecht ist und freie Märkte effizienter funktionieren.

Das ist ein Zirkelschluss und man könnte hier hypothetisch weiterdenken: Wenn allein der Wettbewerb aus ökonomischer Sicht wünschenswert ist und «das Geschäft belebt», wie sprichwörtlich gesagt wird, es in der ökonomischen Rationalität aber einen durch Nutzenmaximierung begründeten Drang zur Konzentration gibt, dann müsste sich Wettbewerb kannibalisieren. Picard (2002) beschreibt aus ökonomischer Perspektive in seiner Spirale nur den umgekehrten Fall: Wenn auf einem Markt die Zahl der Medienanbieter wächst, reduzieren sich automatisch die Nutzerzahlen der einzelnen Angebote. Damit stehen wegen der Werbefinanzierung weniger Ressourcen für die Inhalteproduktion zur Verfügung, was zu einer Verringerung der Qualität und in der Folge auch zu einer weiteren Verringerung der Nutzerzahlen führt (vgl. auch van Cuilenburg, 2007).

Ob sich die Entwicklungen des Printmedienmarktes derart simpel beschreiben lassen und es $\mathrm{zu}$ einem Automatismus hin zu Monopol- oder Oligopolbildung kommt, wie es neomarxistische Autoren annehmen (vgl. Knoche, 2007) kann an dieser Stelle nicht weiterverfolgt werden. Auch die Frage, ob sich die Spirale umkehren lässt und ob damit eine Verringerung der Anbieterzahlen für mehr Ressourcen und im zweiten Schritt mehr Qualität im Markt sorgt, kann nicht beantwortet werden. Hier wäre zumindest anzunehmen, dass die wenigen Anbieter ihre Marktmacht ausnutzen und die noch so hohen Gewinne abschöpfen, wie sich aktuell vermutlich am wissenschaftlichen Publikationsmarkt zeigen liesse. Von Bedeutung für diesen Beitrag und nach Literaturlage unstrittig ist, dass Tendenzen zu voranschreitender Medienkonzentration vorhanden sind (vgl. z. B. Baker, 2007). 
Solche Medienkonzentrationsprozesse reduzieren die Anzahl der Anbieter auf dem jeweiligen Markt. Damit laufen sie einer gesellschaftlich gewünschten möglichst grossen publizistischen Angebotsvielfalt zuwider (vgl. van Cuilenburg, 2007). Knoche geht sogar so weit, dass solche Prozesse «die demokratischen Grundlagen kapitalistischer Wirtschafts- und Gesellschaftssysteme [gefährden], weil sie den propagierten Idealen des Wettbewerbs und der publizistischen Vielfalt real widersprechen" (Knoche, 2007, S. 124). Das Erzielen ökonomischer ${ }^{8}$ sowie publizistischer Vorteile ${ }^{9}$ bei wachsenden Unternehmen und die Gefährdung der Vielfalt stehen konsequenterweise seit Jahren auf der Agenda der internationalen Community (vgl. exemplarisch Baker, 2007) und werden für Deutschland bereits sehr detailliert von Röper (2014) beschrieben.

Aus der Perspektive einiger Wissenschaftler - in jedem Fall aber aus der Perspektive der Medienpolitik muss offenbar eingegriffen werden (vgl. van Cuilenburg, 2007). Die politisch etablierten Lösungen stellen direkte und indirekte sowie allgemeine und selektive Presseförderungsmassnahmen dar, die von Murschetz (2013b) umfassend aufgearbeitet sind. Es stellt sich nun erstens die Frage, ob diese Massnahmen einen Effekt haben. Aus empirischer Perspektive ist es dabei nicht hilfreich von vornherein anzunehmen, dass das nicht der Fall ist (vgl. Murschetz, 2013b). Detaillierter ist interessant, welche Massnahmen welche Effekte haben.

\section{Forschungsdesign und Länderstichprobe ${ }^{10}$}

Für die Erforschung des kausalen Einflusses von Presseförderung auf Medien-

8 Diese entstehen z. B. durch die die Vergrösserung der Ressourcen, des Knowhow und der Leserschaft (vgl. Karstens und Schütte 2010, S. 22-23).

9 Knoche (2007, S. 127) nennt hier «die Konzentration redaktioneller Einheiten sowie [...] die Homogenisierung von Inhalten".

10 Diskussionen und verschiedene Modellierungen der Forschungsdesigns und Länder- konzentration wäre idealiter ein experimentelles Forschungsdesign notwendig, das die Einflüsse von spezifischen Fördermassnahmen in einem Längsschnitt analysiert. Veränderungen in den Medienkonzentrationsraten wären somit unmittelbar kausal auf die Änderungen in den Förderungen zurückzuführen. Da sich die Manipulation der unabhängigen Variable jedoch den forscherischen Möglichkeiten entzieht, ist ein klassischer experimenteller Aufbau ausgeschlossen. Da Presseförderungsmassnahmen in westlichen Demokratien langjährig etabliert sind und Daten hinsichtlich Presseförderungsmassnahmen und -mittel sowie grundlegende Daten zur Kalkulation von Medienkonzentrationsraten vorliegen, ${ }^{11}$ bietet sich ein Ex-post-facto-Verfahren an. Auf diesem Wege lassen sich in einer speziellen Art experimentellen Aufbaus bereits abgeschlossene Prozesse a posteriori ursächlich untersuchen (vgl. z.B. Atteslander, 2010, S. 181; Schnell, Esser, \& Hill, 2013, S. 222).

Sollten sich bei der Beschreibung der Presseförderungsmassnahmen konkrete Änderungen identifizieren lassen, so können diese als «natürliche» Manipulation der unabhängigen Variablen definiert werden. Diese Änderungen lassen wiederum eine Untersuchung hinsichtlich ihres Einflusses auf die abhängigen Variablen zu, z.B. die Medienkonzentration. Schnell und Kollegen (2013) sprechen in diesem Zusammenhang daher von einem «natürlichen Experiment» (S. 220). Weiterhin bedarf es einer Beobachtung des Zusammenhangs im Längsschnitt über mehrere Jahre hinweg, was zweierlei Gründe hat: Erstens besteht Grund zur Annahme, dass Änderungen von Pressefördermassnahmen einen gewissen Wirkungszeitraum benötigen, um etwaige Änderungen hinsichtlich der Medienkonzentration hervorzurufen. Zweitens werden durch einen Aufbau im Längsschnitt und die damit einhergehende Untersuchung des Wirk-

stichproben sind bereits im Detail publiziert (vgl. Kolb \& Oertel, 2016).

11 Auf die Datengrundlage wird später in Kapitel 5 Bezug genommen. 
zusammenhangs in kausaler zeitlicher Reihenfolge die "Anzahl konkurrierender Kausalmodelle [...] erheblich eingeschränkt» (Bortz \& Döring, 2006, S. 519), um die interne Validität des Forschungsdesigns zu verbessern.

Da es sich bei dieser Untersuchung jedoch um eine retrospektive Betrachtung im Ex-post-facto-Verfahren handelt und es ebenfalls unmöglich ist, ein Mediensystem aus seiner mannigfaltigen kulturellen, sozialen, ökonomischen, geografischen (u.a.) Einbettung zu isolieren, ist eine aktive Eliminierung von Drittvariablen nicht möglich. Hinsichtlich der Kontrolle von Drittvariablen bietet sich jedoch die Methode eines internationalen Vergleichs an, welcher die Kontrolle von Störvariablen zumindest passiv gewährleistet. Einen quasi-experimentellen Ansatz in Bezug auf die Überprüfung von Kausalzusammenhängen im internationalen Vergleich schlagen Przeworski und Teune (1970, S. 32-37) mit dem most similar systems design sowie dem most different systems design vor. Das most similar systems design beruht auf der Selektion möglichst ähnlicher Länder, wobei die Annahme zugrunde liegt, dass annähernd gleiche Länder bei gleichen Problemen, annähernd gleiche Lösungsstrategien verfolgen und vorzufindende Unterschiede in diesen Strategien nur auf wenige marginale Unterschiede zurückzuführen sind, welche wiederum als deren Erklärung dienen (vgl. Przeworski \& Teune, 1970, S. 32-34; Wirth \& Kolb, 2003, S. 115-116; Kolb, 2015, S. 93).

Die Logik des most similar systems design invertierend funktioniert das most different systems design, welches auf der Auswahl möglichst unterschiedlicher Länder beruht. Die Grundannahme hier besteht darin, dass beim Vergleich unterschiedlicher Länder bei gleichen Problemen unterschiedliche Strategien der Lösung verfolgt werden dürften. Sind Gemeinsamkeiten in Hinblick auf diese Strategien zu verzeichnen, resultieren diese ausschliesslich aus wenigen Gemeinsamkeiten (vgl. Przeworski \& Teune, 1970, S. 34-37; Kolb, 2015, S. 93; Wirth \& Kolb, 2003, S. 116).
In Bezug auf die Auswahl der zu vergleichenden Länder eröffnen sich somit verschiedene Optionen: Auf der einen Seite kann sich die Selektion hinsichtlich relevanter Merkmale der auszuwählenden Länder auf Gemeinsamkeiten beziehen. Auf der anderen Seite ist es ebenfalls denkbar, Länder miteinander zu vergleichen, welche (u.a.) bezüglich relevanter Merkmale divergieren. Da unterschiedliche Länder sich politisch, kulturell, sozial, wirtschaftlich hochkomplex zusammensetzen, ist es natürlich nicht gegeben identische Länder bzw. "statistische Zwillinge» für einen Vergleich zu finden. Aus diesem Grund ist es unumgänglich diese Komplexität mit Hilfe zusammenfassender Merkmale zu reduzieren. Als relevante Merkmale dieser Art werden in einschlägiger Literatur unter anderem genannt: Grösse (Einwohnerzahl ${ }^{12}$ ), Typologien von Mediensystemen ${ }^{13}$, soziodemografische Merkmale, politische Strukturen, wirt-

12 Die Einwohnerzahl steht hier stellvertretend für die Grösse eines Landes, da es letztlich nicht zielführend ist, die Fläche als Trennkriterium heranzuziehen. Die für diese Arbeit relevante Marktgrösse sowie die wirtschaftliche Kaufkraft eines Landes hängen nicht mit der Fläche sondern mit der Einwohnerzahl zusammen. Dies wird auch in anderen Studien so gehandhabt: Nach Pelinka (2005, S. 175) - leider ohne Begründung oder Beleg - ist die Grenze von Gross- zu Kleinstaat bei 18 Mio. Einwohner verortet.

13 Die Einordnung erfolgt hier nach Hallin \& Mancini (2004), weil die Autoren ihre Typologie weitgehend auf mediale Faktoren aus dem Printmedienbereich (z. B. "newspaper industry"; Hallin \& Mancini, 2004, S. 67) aufgebaut haben, während beispielsweise Blum (2014) auch den Rundfunksektor stark berücksichtigt (aber zu sehr ähnlichen Einordnungen kommt). Man könnte diskutieren, ob die drei bis vier sprachlich getrennten Medienmarktsegmente der Schweiz auch unterschiedlich in die Typologie einzuordnen sind, u. a. weil Hallin und Mancini (2004, S. 67) Frankreich und Italien in einen anderen Typus eingruppieren. Diese Frage wird von Kolb (2015, S. 274) bezüglich der Fernsehmärkte der Schweiz ebenfalls aufgeworfen. Die dort ermittelten Unterschiede aller kleinen Fernsehmärkte sind gering (vgl. Kolb, 2015, S. 278-279), so dass in dieser Studie keine unterschiedliche Typologisierung 
schaftliche Strukturen, Landessprache, geografischer Raum, Einfluss gleichsprachiger Nachbarstaaten (vgl. Aarebrot \& Bakka, 2003, S. 67-68; Hallin \& Mancini, 2004, S. 26; Kleinsteuber, 2003, S. 79-81; Pelinka, 2005, S. 175; Puppis, 2009, S. 1112; Zerback, 2013, S. 284).

Klar ist, dass eine möglichst hohe Anzahl solcher Merkmale zur stärkeren Kontrolle von Drittvariablen führt und sich somit positiv auf die interne Validität auswirkt (vgl. Wirth \& Kolb, 2003, S. 117). Dagegen führt aber jede Erhöhung der Anzahl gleichzeitig zu einer wachsenden Limitierung des Pools an überhaupt vergleichbaren Ländern, da die «Wahrscheinlichkeit steigt, für einen Markt keinen statistischen Zwilling mehr zu finden» (Zerback, 2013, S. 285). ${ }^{14}$ Demnach gilt es, eine Balance zwischen einer angemessenen Anzahl von Merkmalen und der Möglichkeit, vergleichbare Länder zu selektieren, zu finden. In diesem Zusammenhang sprechen Niedermayer und Widmaier auch von einem «modifizierten most similar systems design» (2003, S. 100).

Die Auswahl der Länder dieses Beitrags generiert sich vordergründig auf Basis der Logik des most similar systems design, wobei allein aus der Tatsache, dass es keine eineindeutigen Pärchen geben kann, auch teilweise die Logik des most different systems design zum Tragen kommt. Die folgende Tabelle 1 zeigt in einem Überblick die für diesen Beitrag selektierten Länder. Dahingehend ist zu bemerken, dass über die Auswahl der hier dargestellten Merkmale (Landessprache, Grösse, Typologie des Mediensystems, Einfluss von Nachbarstaaten) hinaus in allen ausgewählten Ländern funktionale Äquivalenz hinsichtlich des politischen Systems (auf dem Pluralismus beruhende Demokratien), des geografischen Raums (Europa) sowie des Wirtschaftssystems (Kapitalismus) vorliegt.

der Schweizer Sprachregionen vorgenommen wird.

14 Zwillinge sind hier im Sinne der untersuchten Merkmale und nicht von eineiigen bzw. nahezu identischen Zwillingen bzw. Ländern zu verstehen.
Ausgehend von der Schweiz, welche als Kleinstaat vier Landessprachen aufweist, bietet sich Belgien als Vergleichsobjekt an. ${ }^{15}$ Mit einer Einwohnerzahl von 11.2 Mio. handelt es sich ebenfalls um einen Kleinstaat. Belgien gehört ebenfalls dem Northern European or Democratic Corporist Model nach Hallin und Mancini (2004, S. 67) an und weist wie die Schweiz mehrere Landessprachen auf, wobei äquivalent zur Schweiz ein Einfluss grosser Nachbarn (Schweiz: Deutschland, Frankreich, Italien; Belgien: Deutschland, Frankreich und etwas kleiner Niederlande) mit den jeweiligen Landessprachen vorliegt (vgl. Tab 1). Das bedeutet natürlich nicht, dass es keine Unterschiede zwischen beiden Ländern gibt oder dass sich diese in allen Belangen sehr ähnlich sind.

Bei der Suche nach vergleichbaren kapitalistisch-demokratischen Kleinstaaten aus dem Raum Europa fallen zuvorderst zwei weitere Länder auf, welche darüber hinaus zueinander ein weiteres Pärchen bilden: Österreich und Irland. Im Vergleich zur Schweiz und Belgien handelt es sich bei diesen Staaten ebenfalls um Kleinstaaten, wobei Österreich der gleichen Mediensystem-Typologie angehört, während Irland nach Hallin und Mancini (2004) dem North Atlantic or Liberal Model zuzuordnen ist. Weiterhin weisen beide Länder im Gegensatz zur Schweiz und Belgien nur eine Landessprache ${ }^{16}$ sowie mit

15 Die Schweiz weist zwar vier Landessprachen auf, jedoch ist die Population der rätoromanisch sprechenden Bevölkerung mit 0.5 Prozent Anteil an der Gesamtbevölkerung (vgl. Bundesamt für Statistik, 2015) als sehr gering einzuschätzen. Es existiert jedoch eine rein rätoromanische Tageszeitung in der Schweiz - La Quotidiana. Ähnliches trifft auf die deutschsprachige Community in Belgien zu: Deren Anteil liegt mit 75716 Einwohnern bei ca. 0.7 Prozent Anteil an der Gesamtbevölkerung Belgiens (vgl. Parliament of the German Community 2015). Äquivalent zur Schweiz existiert eine deutschsprachige Tageszeitung in Belgien - das Grenzecho.

16 Irland weist neben Englisch ebenfalls Irisch als offizielle Landessprache aus. Die Population der Iren, welche im Zuge des Zensus 2011 angibt, täglich Irisch zu sprechen, ist mit einer Zahl von 77185 Personen zu ver- 
Tabelle 1: Auswahlkriterien und Kategorisierung der Untersuchungsländer

\begin{tabular}{|c|c|c|c|c|}
\hline$\underline{\text { Länder }}$ & Landessprache & Grösse & Typologie des Mediensystems & Einfluss von Nachbarstaat \\
\hline Schweiz & $\begin{array}{l}\text { Deutsch, } \\
\text { Französisch, } \\
\text { Italienisch, } \\
\text { Rätoromanisch }\end{array}$ & Kleinstaat & $\begin{array}{l}\text { Northern European or } \\
\text { Democratic Corporatist Model }{ }^{1}\end{array}$ & grosse gleichsprachige Nachbarn \\
\hline Deutschland & Deutsch & Grossstaat & $\begin{array}{l}\text { Northern European or } \\
\text { Democratic Corporatist Model }\end{array}$ & kleiner, gleichsprachiger Nachbar \\
\hline Belgien & $\begin{array}{l}\text { Niederländisch, } \\
\text { Französisch, } \\
\text { Deutsch }\end{array}$ & Kleinstaat & $\begin{array}{l}\text { Northern European or } \\
\text { Democratic Corporatist Mode }{ }^{2}\end{array}$ & grosse gleichsprachige Nachbarn \\
\hline Österreich & Deutsch & Kleinstaat & $\begin{array}{l}\text { Northern European or } \\
\text { Democratic Corporatist Model }\end{array}$ & grosser, gleichsprachiger Nachbar \\
\hline Irland & Englisch, Irisch & Kleinstaat & $\begin{array}{l}\text { North Atlantic or } \\
\text { Liberal Model }\end{array}$ & grosser, gleichsprachiger Nachbar \\
\hline \multicolumn{5}{|c|}{$\begin{array}{l}\text { Quelle: eigene Darstellung: Grösse auf Basis von Pelinka (2005, S. 175); Typologie des Mediensystems auf Basis von Hallin und Mancini (2004, } \\
\text { S. 67); Einfluss von Nachbarstaaten auf Basis von Puppis (2009, S. 11-12). } \\
1 \quad \text { Für eine Diskussion der Einordnung der Schweiz insgesamt vql. Fussnote } 13 .\end{array}$} \\
\hline
\end{tabular}

Deutschland resp. Grossbritannien einen grossen gleichsprachigen Nachbarn als Einflussfaktor auf. Zur Vervollständigung des deutschsprachigen Kulturraums wird auch Deutschland als grosses Land untersucht (vgl. Tab 1).

Durch diese Auswahl können einerseits die gewählten Pärchen miteinander verglichen werden, andererseits bietet sich die Möglichkeit, den Einfluss mehrerer Landessprachen durch den Vergleich der Pärchen resp. der betreffenden Länder einzeln zu untersuchen. Da es nach Niedermayer und Widmaier (2003) einem solchen Forschungsdesign zuträglich ist, eine nicht allzu grosse Auswahl an Ländern zu selektieren, endet die theoriegeleitet systematische Auswahl mit diesen fünf Ländern.

\section{Methode und Operationalisierung(sversuche)}

Die Basis der Daten für diese Untersuchung bildet - wie häufig üblich im Kontext kom-

nachlässigen (vgl. Central Statistics Office, 2012, S. 25) - was bei einer Einwohnerzahl im selben Jahr von 4487000 (vgl. WAN-IFRA, 2015) einen Anteil von etwa 1.7 Prozent an der Gesamtbevölkerung ausmacht - allerdings gibt es keine irischsprachige Tageszeitung. parativer Ansätze (vgl. Kleinsteuber, 2003, S. 83) - eine Sekundäranalyse; im Rahmen dieser Untersuchung die World Press Trends Database der WAN-IFRA (2015). In grossen Teilen liefert die genannte Datenbank Rohdaten (bspw. Einwohnerzahlen, Leser-, Umsatz-, und Auflagenzahlen von Tageszeitungen), welche direkt sowie ebenfalls als Grundlage von Kalkulationen - bspw.: Medienkonzentrationsraten genutzt werden können.

Die Qualität der Datenbank wird im wissenschaftlichen Zusammenhang immer wieder stark kritisiert (vgl. detailliert Kolb \& Oertel, 2016), wobei eigentlich nicht die Qualität an sich zu kritisieren ist, sondern die mangelnde Transparenz derselben: Es können nur Annahmen über die Qualität getroffen werden. Da die Verlegerverbände die Zahlen über ihre Branche national erheben, kann man annehmen, dass sie ein Interesse daran haben könnten, besonders gute Umsatz- oder Reichweitenzahlen zu publizieren. Das wäre aber aus zwei Gründen für den Vergleich nicht sehr problematisch: Erstens hätten alle Verlegerverbände ähnliche Interessen und es ist nicht wahrscheinlich, dass sich ggf. geschönte Daten massiv unterscheiden sollten. Das gilt insbesondere für den Längsschnittvergleich innerhalb eines Landes. Zweitens weist die Methodenliteratur zum internationalen Vergleich 
auf die Möglichkeit hin, ausprägungsorientierte Auswertungen zu unterlassen, und lediglich strukturorientierte Auswertungen vorzunehmen (vgl. Kolb \& Beck, 2011). ${ }^{17}$

Hinzu kommt, dass die Datenbank der WAN-IFRA teilweise Lücken aufweist. Da dies besonders vor dem Jahre $2009 \mathrm{zu}$ Buche schlägt und da zum Zeitpunkt der Untersuchungsdurchführung ausschliesslich Daten bis einschliesslich 2014 vorlagen, ist der hinsichtlich dieses Beitrags definierte Untersuchungszeitraum auf das Intervall von 2009 bis 2014 festgelegt. Sekundäranalysen erlauben aber die Fusionierung verschiedener Datensätze zu einem neuen umfänglicheren und vor allem vollständigeren und valideren Datensatz (vgl. Kolb, 2015, S. 111) und ermöglichen so erst den Vergleich verschiedener Länder im Quer- sowie Längsschnitt (vgl. hierzu auch Schnell et al., 2013, S. 242-244).

Als Quellen hinsichtlich der Validierung, Vervollständigung und des Ausbaus des Datensatzes wurden vor allem Statistische Bundesämter und Ministerien der jeweiligen Länder sowie Jahresabschlussberichte von Unternehmen und Verlegerverbänden verwendet. Weiterhin liefern OECD und Eurostat sowie verschiedene Verbände jeweiliger Länder zur Feststellung der Verbreitung von Werbeträgern (bspw. WEMF, IVW, OJD) teilweise brauchbare Daten.

Für eine konkrete Gegenüberstellung der unabhängigen Variablen (Presseförderungsmassnahmen sowie -mittel) und der abhängigen Variablen (Medienkonzentration) werden im Ergebnisteil in einem ersten Schritt die Fördermassnahmen sowie die Fördermittelhöhe der jeweili-

17 Bei solchen stehen beispielhaft nicht mehr $X$ Euro in Land A und Y Euro in Land B im Zentrum des Vergleichs. Stattdessen wird die Entwicklung von $\mathrm{X}$ auf "Anteil von $\mathrm{X}$ » Euro in Land $\mathrm{A} \mathrm{zu}$ den Zeitpunkten $\mathrm{t}$ und $\mathrm{t}+1$ verglichen mit der Entwicklung von $Y$ Euro auf "Anteil von Y» Euro in Land B zu denselben oder auch anderen Zeitpunkten. Das löst auch Probleme, die beim Vergleich der Schweiz mit Euro-Ländern durch den z.T. stark schwankenden Frankenkurs ausgelöst werden. gen Länder absolut präsentiert (vgl. Kapitel 6.1).$^{18}$ In einem weiteren Schritt werden diese Fördermittel abstrahiert und mittels der im Folgenden erläuterten Förderkoeffizienten dargestellt. Dies bietet den Vorteil der relativen Vergleichbarkeit von Fördermassnahmen anhand von länderspezifischen Kennzahlen (Einwohner-, Leserzahlen, BIP). Diese umfassende Herangehensweise ermöglicht das Ausprobieren verschiedener Messwerte für die Analyse, um herauszuarbeiten, welcher Wert

18 Andere Studien arbeiten insbesondere für die indirekte Pressförderung durch Steuererleichterungen nicht mit den absoluten Zahlen, sondern z. B. mit z-standardisierten Werten, die aus der Reduktion des Steuersatzes in Prozentpunkten errechnet werden (vgl. Brüggemann et al., 2014). Auch durch Reviewerkommentare sind wir auf die Möglichkeit hingewiesen worden, weil diese Vorgehensweise die unterschiedliche Kaufkraft in den absoluten Daten herausnehmen soll. Für eine solche Bereinigung um die Kaufkraftunterschiede werden die Werte in diesem Papier mit dem BIP verrechnet, was u.E. eine transparentere Vorgehensweise darstellt. Zudem ist auch die Verwendung von Prozentpunkten als Basis für die z-Standardisierung fragwürdig, da die verschiedenen Länder unterschiedliche Voraussetzungen haben: So kann die Schweiz z. B. die Mehrwertsteuer gar nicht um 12 Prozentpunkte senken, wie es Deutschland tut, weil die Schweiz nur 8 Prozent Mehrwertsteuer hat. Man könnte noch die anteilige Reduktion berechnen, also z. B. von 19 auf 7 Prozent $=$ Reduktion um gut 63 Prozent in Deutschland, von 8 auf 2.5 Prozent=Reduktion um knapp 69 Prozent in der Schweiz, um eine genauere Erfassung zu bekommen. In diesem Beispiel kehren sich die Verhältnisse sogar um, da Deutschland ja um 12 Prozentpunkte und die Schweiz um 5.5 Prozentpunkte reduziert. Eine validere Erfassung müsste letztlich bestimmen, wie hoch die Anteile der Steuererleichterungen sind, die im jeweiligen Markt auf die Käufer umgelegt werden könnten, und wie hoch der Anteil der vom Unternehmen zu tragenden Mehrkosten wäre, wenn es die Steuererleichterungen nicht gäbe. Diese (Schein-) Genauigkeit führt zum jetzigen Zeitpunkt sicherlich zu weit, zumal es sich immer um Schätzungen oder Übertragungen von Reaktionen auf den Wegfall von Steuererleichterungen in anderen Branchen des jeweiligen nationalen Marktes handeln müsste. 
welche Stärken und welche Schwächen hat, wie das auch in der gängigen Methodenliteratur getan wird (vgl. McDonald \& Dimmick, 2003) und sich für die Analyse von Vielfalt im Fernsehen bewährt hat (vgl. Kolb, 2015)

\subsection{Förderkoeffizient «FK-Leser»}

Der FK-Leser stellt einen Bezug zwischen Marktgrösse und Presseförderungsmitteln her. Als Mittelwert der Förderung in EUR pro (Tageszeitungs-)Leser eines Landes wird der Koeffizient durch die Division der Pressehilfen durch die absolute Leserzahl gebildet. Der Vorteil dieses Koeffizienten ist die direkte Relation der Fördermittel zur tatsächlichen Leserschaft eines Landes. Der Nachteil begründet sich indirekt ebenfalls aus der Leserschaft eines Landes: So steigt der Koeffizient naturgemäss nicht ausschliesslich, je höher die Mittel der Presseförderung ausfallen, sondern ebenfalls, je geringer die Reichweite und damit die Leserzahl eines Landes sind. Es wird also keine besonders gute Brauchbarkeit angenommen.

\subsection{Förderkoeffizient «FK-Einwohner»}

Der FK-Einwohner gibt Aussage über die mittlere Fördersumme in Euro, die ein Staat pro Einwohner ausgibt. Da die Einwohnerzahl eines Staates einen indirekten Bezug zu Staatseinnahmen (Steuern) und Wirtschaftskraft aufweist, stellt die Gewichtung, welche durch die Einwohnerzahl entsteht (je mehr Presseförderungsmittel und je weniger Einwohner, desto höher der FK-Einwohner und vice versa), eine besser realitätsbezogene Gewichtung dar. Liegt die Annahme zugrunde, dass ein Staat mit einer grösseren Population absolut betrachtet mehr Mittel zur Verfügung hat und demnach fähig ist, potentiell mehr Mittel in die Presseförderung $\mathrm{zu}$ investieren, wirkt die diesen Umstand beeinflussende Einwohnerzahl als Grössenunterschied ausgleichend, auch wenn hier noch keine Kaufkraftunterschiede eingehen. Somit bricht der FK-Einwohner die Presseförderungsmittel auf ein länderübergreifendes vergleichbares Mass herunter, was diesen Koeffizienten im Quersowie Längsschnitt interpretierbar macht.
Ein Problem, welches im Zusammenhang mit den Förderkoeffizienten Leser sowie Einwohner hinsichtlich des Querschnittsvergleichs auftritt, ist die abweichende Währung der Schweiz, wobei sich der Schweizer Franken im Untersuchungszeitraum deutlich stärker in Relation zum Euro entwickelte: Ist der Schweizer Franken im Jahr 2009 noch 0.67 EUR wert, so muss im Jahr 2012 bereits 0.83 EUR für einen Schweizer Franken bezahlt werden (vgl. Tabelle A.2). Es wird also nur eine mittlere Brauchbarkeit angenommen.

\subsection{Förderkoeffizient «FK-BIP»}

Der FK-BIP stellt den Anteil der Presseförderungsmittel am Brutto-Inlands-Produkt dar. Da sich eine Darstellung in Prozent im Bereich der zweiten Dezimalstelle befände, wird im Rahmen dieser Untersuchung zugunsten einer angenehmeren Lesbarkeit die Darstellung im Promille-Bereich gewählt.

Da der FK-BIP eine Relation der Fördermittel zur Kaufkraft und somit Wirtschaftskraft eines Landes herstellt, und die Annahme besteht, dass Länder mit hohem BIP mehr Mittel allgemein und damit potentiell mehr Mittel für die Förderung der Presse zur Verfügung haben sollten, erschliesst sich eine optimale Vergleichbarkeit im Querschnitt der Untersuchungsländer. Ein weiterer Vorteil ist, dass der FK-BIP als Anteilskoeffizient losgelöst von Währungen interpretierbar ist. Überdies bietet der FK-BIP im Längsschnittvergleich die Möglichkeit zur Kontrolle, ob ein Land in Relation zu den Vorjahren Potentiale zur Förderung ausnutzte resp. einbüsste. Da die WAN-IFRA alle Daten in "current prices" (bspw. WAN-IFRA, 2010, S. 114) angibt, erschliesst sich die Nutzung des nominellen BIP als Bezugsgrösse.

Für die abhängige Variable Medienkonzentration existieren nach Puppis (2010, S. 275-278) drei etablierte Arten der Messung: die Nennung der Anzahl der Anbieter an einem Markt, der Herfindahl-Hirschmann-Index (HHI) sowie die Berechnung der Concentration 
Ratio. ${ }^{19}$ Die Methode der Nennung der Anzahl an Anbietern auf einem Markt gestaltet sich für einen Vergleich als zu ungenau, da eine absolute Masszahl keine direkte Relation zwischen den einzelnen Ländern zulässt. Darüber hinaus entstünde auch erst durch die erweiterte Interpretation und Gegenüberstellung mit der Anzahl an Tageszeitungen eine wirkliche Relationsebene, welche jedoch nur eine ungenaue Aussage über die tatsächliche Konzentration zuliesse, da keine Information über den Einfluss resp. die Reichweite der jeweiligen Anbieter transportiert wird. So kann einerseits ein Anbieter mit mehreren Tageszeitungen eine kleine Auflage und damit eine geringe Reichweite generieren, andererseits ist es ebenfalls denkbar, dass wenige Anbieter einen grossen Teil des Marktes beherrschen (vgl. Puppis, 2010, S. 275-276).

Der Herfindahl-Hirschmann-Index (HHI) entsteht über die Summierung der quadrierten Marktanteile (Umsatz oder Auflage) jedes marktteilnehmenden Unternehmens. Das Ergebnis ist ein abstrakter Wert, welcher Aussagen über geringe (HHI $\leq 1000)$, mässige $(1000<\mathrm{HHI} \leq 1800)$ resp. hohe $(\mathrm{HHI}>1800)$ Medienkonzentration zulässt (vgl. Heinrich, 2010, S. 123; Napoli, 2001, S. 171; Puppis, 2010, S. 276). Der grosse Vorteil des HHI ist, dass er alle Unternehmen an einem Markt sowie deren Grösse in die Rechnung mit ein-

19 Bei absoluter ökonomischer Medienkonzentration kann es sich neben Fusionen und Übernahmen auch um Joint-Ventures oder Beteiligungen handeln. Daher ist zusätzlich festzulegen, wie die Zuordnung in diesen Fällen erfolgt. Da es in dieser Untersuchung letztlich um Aussagen über inhaltliche Vielfalt geht, sowie die Annahme besteht, dass sich ökonomischer Einfluss in inhaltlichem Einfluss widerspiegelt (vgl. Knoche, 2007, S. 124), wird bei einer Beteiligung eines Medienunternehmens an einer Tageszeitung ab über 50 Prozent die komplette Auflage dieser Tageszeitung dem betreffenden Medienunternehmen zugeschrieben. Bei Beteiligungen mehrerer Medienunternehmen ohne eine Partei mit eben beschriebener Mehrheit wird die Auflage der entsprechenden Tageszeitungen den Anteilen der jeweiligen Medienunternehmen entsprechend verteilt. bezieht. Der Nachteil ist, dass man dafür auch belastbare Daten für alle Unternehmen benötigt und dass die Werte nicht direkt eingängig und interpretierbar sind.

Die dritte Möglichkeit, die Medienkonzentration zu messen, bietet sich mit der Concentration Ratio (CR; folgend auch Konzentrationsrate). Für diesen Beitrag wird diese Methode angewendet, was sich aus zwei Gründen erklärt: Erstens bietet die Datenlage der WAN-IFRA nicht die Möglichkeit, Medienkonzentrationswerte mittels des HHI zu bestimmen, da keine Vollständigkeit der Anbieter und Marktanteile bis zu den kleinsten Marktteilnehmern im Bereich Tageszeitungen gegeben ist. Zweitens erschliessen sich CR-Werte in ihrer Form als Prozentwerte direkt logisch und müssen nicht wie der HHI abstrakt interpretiert werden. Die Konzentrationsrate bezieht sich ebenfalls auf die unabhängigen Anbieter eines Marktes, misst über die erste Methode hinaus jedoch deren Marktanteil in Abhängigkeit von Auflage oder Umsatz. Angegeben werden CR-Werte als CR3, CR5, CR7 (u.a.), wobei sich die Zahl auf die jeweilig grössten - in diesem Fall drei, fünf oder sieben - voneinander unabhängigen Anbieter bezieht. Ein CR3 von 42 würde demnach aussagen, dass die drei auflagen- oder umsatzstärksten unabhängigen Anbieter von Tageszeitungen 42 Prozent der Auflage resp. des Umsatzes in Relation zur Gesamtauflage resp. zum Gesamtumsatzes des Tageszeitungsmarktes auf sich vereinen (vgl. Dreier, 2006, S. 185; Heinrich, 2010, S. 122-123; Puppis, 2010, S. 276). Nach Ward (2004, S. 8) gilt ein CR3-Wert bis 35 als niedrige (low) Konzentration, im Bereich der Werte über 35 bis 55 als mässige (moderate) Konzentration sowie ab einem CR3-Wert grösser als 55 als hohe (high) Konzentration. Da die Auflagenzahl einen direkteren Bezug zur Anzahl der Leser hat als die Umsatzzahl eines Medienunternehmens, somit eine genauere Aussage über den inhaltlichen Einfluss eines Medienunternehmens ergibt, ist die Bezugsebene der in dieser Untersuchung 
betrachteten CR-Werte die Auflage von Tageszeitungen. ${ }^{20}$

\section{Ergebnisse}

Im Folgenden wird kurz auf die charakteristischen Merkmale jedes Landes hinsichtlich der etablierten Pressefördermassnahmen sowie die jeweiligen Fördermittelhöhen eingegangen. Die Schweiz fährt im Hinblick auf eine indirekte allgemeine Presseförderung zweigleisig: Neben der Minderung des Steuersatzes auf Presseartikel installierte die Schweiz eine weitere Fördermassnahme in Form einer Abgabe seitens des Staates an die Schweizer Post, welche dadurch Transportkosten für Zeitungen mit weniger als 40000 Exemplaren preiswerter anbieten kann, womit die Distributionskosten der Unternehmen verringert werden. Zum Jahr 2012 wurde diese Abgabe von einem bis dahin jährlich festen Wert von 30 Mio. CHF auf 50 Mio. CHF angehoben (vgl. Hugentobler \& Jaag, 2013; WAN-IFRA, 2010, S. 424; Tabelle 2). Damit wächst die Presseförderung um ca. 30 Prozent an, was sich als eine quasi-experimentelle Ursache innerhalb eines Landes anbietet.

Das ebenfalls mehrsprachige Belgien ist neben Grossbritannien das einzige Land der Europäischen Union, welches nicht nur einen verminderten Mehrwertsteuersatz auf Presseartikel erhebt, sondern vielmehr gänzlich auf die Erhebung einer Mehrwertsteuer verzichtet. Über diese allgemeine indirekte Massnahme hinaus fördert Belgien selektiv direkt die deutsch- und französischsprachigen Ta-

20 Des Weiteren erfolgt an dieser Stelle die Eingrenzung auf die Betrachtung von CR3-Werten: Da im Hinblick auf Kleinstaaten durch die geringere Einwohnerzahl eine kleinere absolute Marktgrösse sowie (ebenfalls dadurch bedingt) eine eher geringe Anzahl an Titeln und Anbietern vorliegt, bietet der CR3Wert eine potentiell bessere Möglichkeit der Vergleichbarkeit der Untersuchungsländer. CR5-Werte zeigen sich als teilweise zu gross, so weist bspw. Belgien im Jahr 2012 bereits einen CR5-Wert von 96.8 auf (vgl. Oertel, 2014, S. A6), so dass Deckeneffekte die Analysen erschweren würden. geszeitungen (vgl. Tabelle 3). Für 2013 und 2014 weist die Datenbank leider keine Werte aus. Aus den aktuelleren Werten für 2015 bzw. 2016 lässt sich aber rückschliessen (vgl. Marcourt, 2016; Regierung der Deutschsprachigen Gemeinschaft Belgiens, 2016), dass die direkte Förderung relativ stabil um 8 Millionen Euro geblieben sein dürfte. Der Rückgang um etwa ein Viertel von 2011 auf 2012 und die Folgejahre kann ebenfalls genauer analysiert werden, auch wenn dieser nur auf den Umsatzrückgang zurückgeht.

Wie Tabelle 4 zeigt, verfügt Österreich im Vergleich zu den anderen Untersuchungsländern über ein breiteres Spektrum an Presseförderungsmassnahmen. Zuvorderst sei die reduzierte Mehrwertsteuer auf Presseartikel genannt (vgl. Murschetz \& Karmasin, 2013, S. 139; WAN-IFRA, 2010, S. 136). Im Untersuchungszeitraum zeigen sich der verminderte sowie der Normalmehrwertsteuersatz konstant bei 10 resp. 20 Prozent. An dieser Stelle sei jedoch erwähnt, dass Österreich sich mit einem verminderten Mehrwertsteuersatz von 10 Prozent auf Presseartikel deutlich im oberen Bereich der höchsten Steuersätze auf Presseartikel in der Europäischen Union befindet (vgl. Murschetz \& Karmasin, 2013, S. 139). Die sich durch die Mehrwertsteuerreduktion ergebenden Fördersummen zeigen sich im Zeitraum von 2009 bis 2014 von ca. 15.9 Mio. EUR im Jahr 2009 auf 12.9 Mio. EUR im Jahr 2014 als rückläufig, was sich anhand der im gleichen Zeitraum rückläufigen Auflage bezahlter Tageszeitungen sowie damit einhergehender Umsatzrückgänge erklären lässt (vgl. Tabelle 4).

Neben der Reduktion der Mehrwertsteuer sind in Österreich weitere allgemein indirekte sowie selektiv direkte Fördermassnahmen etabliert. Als allgemein indirekte Förderung vergibt Österreich Hilfen an Tageszeitungen, um den Vertrieb zu unterstützen. Wie Tabelle 4 zeigt, liegt diese Summe über die Jahre 2009 und 2010 konstant bei 4.5 Mio. EUR, während in den Jahren 2011 bis 2014 ein schrittweises Sinken dieser Summe zu verzeichnen ist. Weiterhin findet eine Qualitätsförderung in Form von Journalistentrainings statt. Die- 
Tabelle 2: Presseförderungsmassnahmen Schweiz 2009 bis 2014 (CHF)

\begin{tabular}{lcccccr}
\hline Massnahme & 2009 & 2010 & 2011 & 2012 & 2013 & 2014 \\
\hline $\begin{array}{l}\text { Allgemein indirekt } \\
\text { verminderte MWST' }\end{array}$ & 32348 & 32958 & 34663 & 34395 & 34073 & 34288 \\
$\begin{array}{l}\text { Selektiv indirekt } \\
\quad \text { Swiss Post }\end{array}$ & & & & & & \\
Gesamt & 30000 & 30000 & 30000 & 50000 & 50000 & 50000 \\
\hline
\end{tabular}

Quelle: eigene Darstellung.

1 Kalkulation mittels der jeweilig geltenden MWST und der Umsätze aus Verkäufen von Tageszeitungen (WAN-IFRA, 2015). Berechnungsbeispiel: In der Schweiz gelten generell $8 \%$ Mehrwertsteuer, auf Presseartikel aber nur 3\%. Berechnet wird die Differenz aus den potenziell erreichten Bruttoumsätzen (inkl. $8 \%$ MWST) und den tatsächlichen Bruttoumsätzen (inkl. 3\% MWST).

2 WAN-IFRA (2015).

Tabelle 3: Presseförderungsmassnahmen Belgien 2009 bis 2014 (EUR)

\begin{tabular}{lrrrrrr}
\hline Massnahme & 2009 & 2010 & 2011 & 2012 & 2013 & 2014 \\
\hline $\begin{array}{l}\text { Allgemein indirekt } \\
\quad \text { verminderte MWST }\end{array}$ & 70350 & 73500 & 77280 & 57960 & 56995 & 56545 \\
$\begin{array}{l}\text { Selektiv direkt } \\
\quad \text { frankophone und deutschsprachige Tageszeitungen }{ }^{2}\end{array}$ & 6900 & $7130^{3}$ & 7360 & $7476^{3}$ & 7591 & 7790 \\
Gesamt & 77250 & 80630 & 84640 & 65436 & 64586 & 64335 \\
\hline
\end{tabular}

Quelle: eigene Darstellung.

1 Kalkulation mittels der jeweilig geltenden MWST und der Umsätze aus Verkäufen von Tageszeitungen (WAN-IFRA, 2015).

2 WAN-IFRA (2010, S. 149; 153; 2015), E-Mail Anfragen beim Centre de l'aide à la presse écrite.

3 Für die Jahre 2010 und 2012 liegen keine Wert vor, daher erfolgt die Schätzung dieses Wertes aufgrund der folgenden Steigerung dieser Fördermassnahme als Mittelwert zwischen den Werten von 2009 und 2011 bzw. 2011 und 2013.

Tabelle 4: Presseförderungsmassnahmen Österreich 2009 bis 2014 (EUR)

\begin{tabular}{|c|c|c|c|c|c|c|}
\hline Massnahme & 2009 & 2010 & 2011 & 2012 & 2013 & 2014 \\
\hline \multicolumn{7}{|l|}{ Allgemein indirekt } \\
\hline verminderte MWST ${ }^{1}$ & 15948 & 13900 & 13817 & 13907 & 13079 & 12910 \\
\hline Qualitätsförderung² & 1668 & 1668 & 1608 & 1575 & 1560 & 1532 \\
\hline Vertriebsförderung ${ }^{2}$ & 4525 & 4525 & 4362 & 3924 & 3885 & 3710 \\
\hline Gesamt & 22141 & 20093 & 19787 & 19406 & 18524 & 18152 \\
\hline \multicolumn{7}{|l|}{ Selektiv direkt } \\
\hline besondere Förderung ${ }^{2}$ & 6645 & 6645 & 6406 & 5287 & 5242 & 3242 \\
\hline Gesamt & 28786 & 26738 & 26193 & 24693 & 23766 & 21394 \\
\hline \multicolumn{7}{|l|}{ Quelle: eigene Darstellung } \\
\hline \multicolumn{7}{|c|}{$\begin{array}{l}1 \text { Kalkulation mittels der jeweilig geltenden MWST und der Umsätze aus Verkäufen von Tageszeitungen (WAN-IFRA, 2015). Aufgrund des } \\
\text { Mangels an Daten des Umsatzes aus Verkäufen von Tageszeitungen für die Jahre } 2009 \text { und } 2010 \text { werden diese geschätzt (vgl. Anhang: } \\
\text { Tab A.1) }\end{array}$} \\
\hline
\end{tabular}

se Massnahme ist universell zugänglich, daher erfolgt die Einordnung ebenfalls unter allgemein direkter Förderung. Analog zur Vertriebsförderung erfuhr die Qualitätsförderung nach einer konstanten Pha- se von 2009 bis 2010 von 1.668 Mio. EUR ein stufenweises Sinken auf 1.608 Mio. EUR im Jahr 2011 sowie 1.532 Mio. EUR im Jahr 2014. Des Weiteren vergibt Österreich eine besondere Förderung an be- 
Tabelle 5: Presseförderungsmassnahmen Irland 2009 bis 2014 (EUR)

\begin{tabular}{lcccccr}
\hline Massnahme & 2009 & 2010 & 2011 & 2012 & 2013 & 2014 \\
\hline $\begin{array}{l}\text { Allgemein indirekt } \\
\text { verminderte MWST }\end{array}$ & 23084 & 20352 & 24203 & 34221 & 33212 & 32393 \\
\hline
\end{tabular}

Quelle: eigene Darstellung; Kalkulation mittels der jeweilig geltenden MWST und der Umsätze aus Verkäufen von Tageszeitungen (WAN-IFRA, 2015).

Tabelle 6: Presseförderungsmassnahmen Deutschland 2009 bis 2014 (EUR)

\begin{tabular}{lrrrrrr}
\hline $\begin{array}{l}\text { Massnahme } \\
\text { Allgemein indirekt }\end{array}$ & 2009 & 2010 & 2011 & 2012 & 2013 & 2014 \\
$\begin{array}{l}\text { verminderte MWST } \\
\text { Quelle: eigene Darstellung; Kalkulation mittels der jeweilig geltenden MWST und der Umsätze aus Verkäufen von Tageszeitungen: } 2006\end{array}$ \\
$\begin{array}{l}\text { (BDZV, 2007), 2007-2012 (WAN-IFRA, 2015). }\end{array}$
\end{tabular}

zahlte Tageszeitungen, welche weniger als 100000 Exemplare verkaufen, womit die Intention der Sicherung struktureller Vielfalt durch den Ausschluss der Marktführer verfolgt wird. Diese Förderungsmassnahme ist als selektiv direkt kategorisiert (vgl. Tabelle 4; sowie auch Murschetz \& Karmasin, 2013, S. 139-144; Statistik Austria, 2013; WAN-IFRA, 2010, S. 137, 2015).

Hinsichtlich Österreichs Presseförderung existiert eine Besonderheit, welche in der regierungsseitigen Schaltung von Anzeigen in Tageszeitungen besteht. Da diese willkürlich und intransparent von den Ministerien geschaltet werden, kann in diesem Kontext über eine mögliche Abhängigkeit der Presse von staatlichen Institutionen gesprochen werden. Dies wiederum birgt ein gesteigertes Potential für einen wohlwollenden Journalismus, Haas spricht in diesem Kontext auch von «Hofberichterstattung» (2012, S. 43). Das widerspricht direkt den Idealen einer Demokratie und der notwendigen strikten Trennung von Staat und Medien. Im Kontext dieser Arbeit sei diese Art der «Unterstützung der Presse» zwar genannt, da sie diffus und intransparent erfolgt, ist sie jedoch schwer bis nicht zu quantifizieren und wird nicht mit in die Liste der Presseförderungsarten und -mittel aufgenommen (vgl. auch Murschetz \& Karmasin, 2013, S. 145; Trappel \& Zettl, 2013).

Irland greift ausschliesslich auf die Fördermassnahme des verminderten
Mehrwertsteuersatzes auf Presseartikel zurück (vgl. WAN-IFRA, 2010, S. 266). Im Untersuchungszeitraum von 2009 bis 2012 passte Irland die Steuersätze vermehrt an, so dass sich in den Werten der Tabelle 5 trotz rückläufigen Umsatz- und Auflagezahlen (vgl. Tabelle A.4) eine Steigerung der Fördersummen abzeichnet. Wobei hier abermals darauf hinzuweisen ist, dass es sich nicht um Geld handelt, was den Medienunternehmen zugutekommt, sondern vielmehr um Geld, was die Unternehmen im Vergleich zur Normalbesteuerung nicht zusätzlich aufbringen müssen. Hier kann der Sprung um gut 40 Prozent von 2011 auf 2012 genauer analysiert werden.

Deutschland fördert - genau wie Irland - ausschliesslich indirekt und allgemein mittels des verminderten Mehrwertsteuersatzes. Im Verlauf der Jahre von 2009 bis 2013 ist dahingehend ein kontinuierlicher Rückgang zu verzeichnen, 2014 zeigt sich ein leichter Anstieg im Vergleich zu 2013 (vgl. Tabelle 6). Die Entwicklungen sind erneut mit der Entwicklung der Auflagenzahlen (vgl. Tabelle A.4) im gleichen Zeitraum zu erklären.

Hinsichtlich des FK-Einwohner ist vor allem Österreich in der Gruppe der Kleinstaaten auffällig, da hier mit sogar sinkender Tendenz von 2009 zu 2014 mit Abstand am wenigsten EUR pro Einwohner für die Presseförderung ausgegeben werden. Umgekehrt liegt Belgien bis 2011 deutlich über dem Durchschnitt und investiert in 
Tabelle 7: FK-Einwohner (EUR pro Einwohner)

\begin{tabular}{llcccccc}
\hline Land & & 2009 & 2010 & 2011 & 2012 & 2013 & 2014 \\
\hline Schweiz & EUR $^{1}$ & 5.40 & 6.39 & 6.68 & 8.69 & 8.08 & 8.22 \\
& (CHF) & $(8.01)$ & $(8.00)$ & $(8.13)$ & $(10.50)$ & $(9.92)$ & $(9.88)$ \\
Belgien & 7.18 & 7.41 & 7.69 & 5.87 & 5.77 & 5.73 \\
Österreich & 3.45 & 3.19 & 3.11 & 2.92 & 2.80 & 2.51 \\
Irland & 5.16 & 4.54 & 5.39 & 7.46 & 7.23 & 7.02 \\
Mittelwert Kleinstaaten & & 5.30 & 5.38 & 5.72 & 6.24 & 5.97 & 5.87 \\
\hline Deutschland & 10.91 & 11.00 & 10.98 & 10.59 & 10.13 & 10.25 \\
\hline
\end{tabular}

Quelle: eigene Darstellung; Kalkulation (s. Kapitel 2.4.1) auf Basis der im Zuge des Kapitels 3.1 dargestellten Werte.

1 Die Werte der Schweiz in EUR basieren auf der Umrechnung anhand des Wechselkurses des 31.12. des jeweiligen Jahres (vgl. Tabelle A.2).

2 Der Mittelwert bezieht sich ausschliesslich auf die Kleinstaaten. Hinsichtlich der Schweiz fliesst der Wert in EUR pro Einwohner in die Berechnung mit ein.

der Gruppe der Kleinstaaten am meisten EUR pro Kopf. Zum Ende des Beobachtungszeitraumes überholen Irland und die Schweiz Belgien hinsichtlich des FK-Einwohner, was sich für die Schweiz einesteils aus dem Erstarken des Schweizer Franken erklären lässt, anderenteils aber auch aus der Anhebung der Förderung mittels der Swiss Post (vgl. Tabelle 3). ${ }^{21}$ Irland hingegen passt im Zuge der Wirtschaftskrise in diesem Zeitraum mehrfach die Mehrwertsteuersätze an und senkt als einziges der Untersuchungsländer im Beobachtungszeitraum den verminderten Mehrwertsteuersatz auf Presseartikel, wodurch sich im Endeffekt die Steigerung des FK-Einwohner erklären lässt (vgl. Tabelle 7). Damit lassen sich für die nationalen Betrachtungen erneut die Jahre 2011 und folgende als besonders interessant herausarbeiten: In der Schweiz und in Irland gibt es sprunghafte Zuwächse, die sich in der Folge auch in den Konzentrationsmessungen niederschlagen könnten.

Der Vergleich mit dem Grossstaat Deutschland zeigt jedoch eine enorme Lücke im Hinblick auf die Höhe der Werte

$21 \mathrm{Ob}$ durch die Erhöhung des Etats zur Förderung der Zustellung von Printmedien in der Schweiz tatsächlich und/oder ausschliesslich die Medien gefördert werden, kann zumindest diskutiert werden, zumal die Post nach Angaben eines Reviewers gleichzeitig die Gebühren angehoben haben sollen. Vielen Dank für den Hinweis, einen wissenschaftlich belastbareren Beleg dafür konnten die Autoren allerdings nicht finden. des FK-Einwohner, so weist Deutschland im Längsschnitt zwar eine leicht sinkende Tendenz von 2009 zu 2014 auf, jedoch liegt es mit Werten zwischen 10.13 EUR und 11.00 EUR pro Einwohner deutlich über den Werten der Kleinstaaten (vgl. Tabelle 7). Das Ergebnis ist wenig überraschend: Durch grosse Märkte können höhere Umsatzzahlen generiert werden, die auch zu höheren Beträgen durch reduzierte Mehrwertsteuersätze führen. Seit 2012 fallen die Werte fast durchgängig in allen Ländern, weil die Auflagenzahlen sinken und damit auch die Mehrwertsteuer-Reduktionen geringer ausfallen. Damit ist der Wert insgesamt leider - wie angenommen - wenig aussagekräftig für die weitere Analyse.

Die Betrachtung der Fördermittel pro Leser ist unabhängiger von der Marktgrösse als der FK-Einwohner. Die Länder zeigen folglich in Relation zu den Tageszeitungslesern andere Werte als in Bezug auf die Einwohner (vgl. Tabelle 8).

Bezüglich des FK-Leser ist zuvorderst abermals Österreich zu bemerken, welches auch in Bezug auf die Förderung pro Leser mit sinkender Tendenz und Werten von 5.43 EUR bis 4.27 EUR im Beobachtungszeitraum ebenfalls die mit grossem Abstand geringsten Werte aufweist. Die Werte der restlichen Kleinstaaten bewegen sich in ähnlichem Rahmen über dem Durchschnitt. Was in Relation zum FK-Einwohner abweicht, ist jedoch der Vergleich mit dem Grossstaat Deutschland: Weist Deutschland in Bezug auf die Fördermittel pro Einwohner noch deutlich höhere Wer- 
Tabelle 8: FK-Leser (EUR pro Leser)

\begin{tabular}{|c|c|c|c|c|c|c|c|}
\hline \multicolumn{2}{|l|}{ Land } & 2009 & 2010 & 2011 & 2012 & 2013 & 2014 \\
\hline \multicolumn{2}{|l|}{ Schweiz } & $\begin{array}{c}8.39 \\
(12.45)\end{array}$ & $\begin{array}{c}10.20 \\
(12.77)\end{array}$ & $\begin{array}{c}10.66 \\
(12.96)\end{array}$ & $\begin{array}{c}13.81 \\
\text { (16.68) }\end{array}$ & $\begin{array}{c}15.51 \\
(19.05)\end{array}$ & $\begin{array}{c}16.58 \\
(19.93)\end{array}$ \\
\hline \multicolumn{2}{|l|}{ Belgien } & 15.56 & 15.64 & 16.90 & 12.89 & 13.42 & 14.06 \\
\hline \multicolumn{2}{|c|}{ Österreich } & 5.43 & 5.11 & 5.02 & 4.73 & 4.58 & 4.27 \\
\hline \multicolumn{2}{|l|}{ Irland } & 11.19 & 10.81 & 13.39 & 16.15 & 14.81 & 16.59 \\
\hline \multicolumn{2}{|c|}{ Mittelwert Kleinstaaten $^{2}$} & 10.14 & 10.44 & 11.49 & 11.90 & 12.08 & 12.88 \\
\hline \multicolumn{2}{|c|}{ Deutschland } & 13.77 & 13.86 & 13.85 & 13.39 & 12.65 & 12.84 \\
\hline \multicolumn{8}{|c|}{$\begin{array}{l}1 \text { Die Werte der Schweiz in EUR basieren auf der Umrechnung anhand des Wechselkurses des 31.12. des jeweiligen Jahres (vgl. Ta- } \\
\text { belle A.2). }\end{array}$} \\
\hline \multicolumn{8}{|c|}{ ttelwert bezieht sich ausschliesslich auf die Kleinstaaten. Hinsichtlich der Schweiz fliesst der Wert in EUR pro Einwohner in die } \\
\hline
\end{tabular}

Tabelle 9: FK-BIP (Anteil am BIP in Promille)

\begin{tabular}{lcccccc}
\hline Land & 2009 & 2010 & 2011 & 2012 & 2013 & 2014 \\
\hline Schweiz & 0.17 & 0.15 & 0.14 & 0.17 & 0.13 & 0.13 \\
Belgien & 0.23 & 0.23 & 0.23 & 0.17 & 0.16 & 0.16 \\
Österreich & 0.11 & 0.09 & 0.09 & 0.08 & 0.07 & 0.07 \\
Irland & 0.14 & 0.13 & 0.15 & 0.21 & 0.19 & 0.17 \\
Mittelwert Kleinstaaten ${ }^{21}$ & 0.16 & 0.15 & 0.15 & 0.16 & 0.14 & 0.13 \\
\hline Deutschland & 0.38 & 0.36 & 0.35 & 0.33 & 0.29 & 0.29 \\
\hline
\end{tabular}

Quelle: eigene Darstellung; Kalkulation (s. Kapitel 2.4.1) auf Basis der im Zuge des Kapitels 3.1 dargestellten Werte.

1 Der Mittelwert bezieht sich ausschliesslich auf die Kleinstaaten. Hinsichtlich der Schweiz fliesst der Wert in EUR pro Einwohner in die Berechnung mit ein.

te als die Kleinstaaten auf, so liegt Deutschland beim FK-Leser zwar über dem Durchschnitt der Kleinstaaten - was sich aus der Natur des arithmetischen Mittels durch die geringen Werte Österreichs als niedrig zeigt - aber mit Werten von 12.65 EUR bis 13.86 EUR pro Leser teilweise sogar unter den spezifischen Werten der Kleinstaaten. In den Jahren 2009 bis 2011 liegt Belgien mit Werten zwischen 15.56 EUR und 16.90 EUR pro Leser merklich über den Werten Deutschlands. Im Jahr 2012 bspw. liegen Belgien und die Schweiz in etwa im gleichen Bereich wie Deutschland, jedoch weist Irland mit 16.15 EUR pro Leser einen um 2.76 EUR höheren Wert als Deutschland auf. Die Sprünge von 2011 auf 2012 zeigen sich erneut in Belgien, Irland und der Schweiz (vgl. Tabelle 8).

Die Betrachtung des FK-BIP ändert das bisher gezeichnete Bild nicht, es bestätigt jedoch auch in Bezug auf die Fördermittelhöhe in Relation zum BIP, dass Österreich mit Abstand am wenigsten in die Presseförderung investiert. Im Jahr 2014 ist der FK-BIP Österreichs (0.07) etwas mehr als halb so groß wie der Wert der Schweiz (0.13) und relativ noch einmal kleiner im Vergleich zu Irland (0.17). Im Vergleich zu Deutschland sind die Werte Österreichs über den Beobachtungszeitraum sogar ungefähr viermal kleiner. Wie bereits in Abschnitt 5.3 erklärt, lässt sich dies nicht durch die Grösse erklären, da es sich um einen Relationskoeffizienten handelt, welcher indirekt die Grösse des Landes mit einbezieht (vgl. Tabelle 9).

Die Werte der Schweiz zeigen sich im Zeitraum 2009 bis 2011 von 0.17 auf 0.14 sinkend. Durch die Aufstockung des SwissPost-Etats reguliert sich der Wert zum Jahr 2012 jedoch wieder nach oben auf den Wert des Jahres 2009 (0.17), um danach wieder auf 0.13 abzufallen. Weiterhin fällt abermals der starke Anstieg des FK-BIP Irlands im Jahr 2012 auf, was einesteils auf der bereits im Zuge der Interpretation der Tabelle 7 erwähnten Senkung des vermin- 
derten Mehrwertsteuersatzes - und damit Steigerung der vermeintlichen Fördermittelhöhe-beruht, anderenteils jedoch auch mit dem Sinken des BIP Irlands (vgl. Tabelle A.3) im Untersuchungszeitraum zusammenhängt. Die Werte sinken bis 2014 aber wieder deutlich ab (vgl. Tabelle 9).

Bei Vergleich der Kleinstaaten mit Deutschland zeigt sich, dass Deutschland den mit Abstand höchsten FK-BIP aufweist. Auch wenn die Tendenz von 2009 (0.38) zu 2014 (0.29) als sinkend zu beschreiben ist, sind die Werte in Relation zum Mittelwert der Kleinstaaten kontinuierlich als doppelt so hoch gekennzeichnet (vgl. Tabelle 9). Es scheint sich zu bestätigen, dass für eine Typologie der Presseförderung (oder gar der Mediensysteme) die Marktgrösse in jedem Fall einbezogen werden sollte.

Tabelle 10 zeigt in einem Überblick die CR3-Werte der Untersuchungsländer. Zur Interpretation werden ebenfalls Marktanteile der einzelnen Medienunternehmen herangezogen, welche sofern möglich auf der Kalkulation auf Basis der Daten der WAN-IFRA (2015) beruhen.

Die Schweiz ist im Untersuchungszeitraum vor allem durch die Übernahme der Edipresse Suisse durch die Tamedia AG gekennzeichnet. Diese kauft sich zwar bereits 2009 in Edipresse Suisse ein, hält jedoch erst ab 1. Januar 2011 genau 50.1 Prozent der Anteile; zum 1. Januar 2012 gilt die komplette Übernahme (vgl. Tamedia AG, 2014). Im Jahr 2010 übernimmt die Tamedia AG zusätzlich noch die Zürcher Landzeitung von der NZZ
Medien AG sowie die Zürichsee Zeitung (vgl. NZZ Mediengruppe, 2010). Aus diesen Übernahmen erklärt sich somit auch der Anstieg des CR3-Wertes um 4.9 Prozentpunkte von 69.3 im Jahre 2009 auf 74.2 Prozent im Jahre 2011. Zum Jahre 2012 steigt der CR3-Wert nur leicht um 0.5 Prozentpunkte auf einen Wert von 74.7. Im Jahr 2012 beansprucht allein die Tamedia AG einen Marktanteil von 45.1 Prozent für sich; im Jahr 2009 lag dieser Wert noch bei 39.9 Prozent. Die drei umsatzstärksten Anbieter von Tageszeitungen teilen sich also 2013 (relativ stabil seit drei Jahren) etwa drei Viertel des Marktes (vgl. Tabelle 10; sowie WAN-IFRA, 2015). Das ist ursächlich kaum auf die gestiegene Presseförderung zurückzuführen. Es bleibt zu untersuchen, ob der Rückgang in 2014 eine Auswirkung der Förderung sein könnte.

In Belgien vollzieht sich im Untersuchungszeitraum 2009 bis 2012 hinsichtlich der drei grössten Medienunternehmen im Bereich Tageszeitungen - Concentra, Corelio, De Persgroep - weder positives noch negatives externes Wachstum. Ebenfalls im Hinblick auf internes Wachstum treten weder negative noch positive nennenswerte Veränderungen auf. Von 2009 zu 2010 findet ein Rangfolgenwechsel der zwei grössten Medienunternehmen aufgrund marginaler Auflagenverschiebungen statt: Während Concentra 2010 im Vergleich zum Vorjahr leicht an Auflage verliert und 0.1 Prozentpunkte an Marktanteil verliert, kann Corelio im gleichen Zeitraum leicht zulegen und steigert damit seinen Marktanteil um 0.2 Prozent-

Tabelle 10: Pressekonzentration (CR3)

\begin{tabular}{lcccccr}
\hline Land & 2009 & 2010 & 2011 & 2012 & 2013 & 2014 \\
\hline Schweiz & 69.3 & - & 74.2 & 74.7 & 74.5 & 72.5 \\
Belgien & 76.6 & 76.9 & 78.0 & 79.9 & 79.4 & 79.7 \\
Österreich & 65.9 & 65.0 & 68.2 & 64.4 & 77.9 & 75.2 \\
Irland & 61.8 & 62.7 & 66.1 & 65.6 & 63.5 & $65.7 / 74.21$ \\
\hline Deutschland & - & 34.0 & - & 33.7 & - & 32.7 \\
\hline
\end{tabular}

Quelle: eigene Darstellung; Eigenkalkulation auf Basis der WAN-IFRA (2010; 2015), Ausnahmen: Werte Deutschland (Röper, 2010, S. 222; 2012, S. 273; 2014: 259), weil die Angaben in der WAN-IFRA die Kalkulation nicht erlauben. Die Berechnungsgrundlage ist aber identisch: Reichweite der drei umsatzstärksten Unternehmen auf dem Tageszeitungsmarkt. Werte Belgien 2013 und 2014 (vgl. Centre d'Information sur les Médias, 2015); Werte Schweiz 2014 (vgl. Verband Schweizer Medien, 2014; WEMF, 2014).

1 Am 19. Dezember 2014 wurde der Metro Herald eingestellt (vgl. Molloy, 2014). Der Jahreswert mit der Zeitung ist 65.7\%, ohne die eingestellte Zeitung kommt Irland auf $74.2 \%$. 
punkte. Da beide Konkurrenten nahezu gleiche Auflagenzahlen aufweisen, führt bereits diese kleine Veränderung zum Rangfolgenwechsel, so dass ab 2010 Corelio den ersten Platz innehat. Die Gesamtauflage der Tageszeitungen Belgiens sinkt über den betrachteten Zeitraum von 2009 zu 2012 um 4.3 Prozent, was bei nahezu gleichbleibender Auflage der drei grössten Medienunternehmen mathematisch zu einem steigenden CR3-Wert führt: Im Jahr 2009 liegt der CR3 bei 76.6 Prozent, im Jahr 2010 steigt dieser auf 76.9 Prozent, im Jahr 2011 auf 78 Prozent und 2012 auf den Wert von 79.9 Prozent. In der Folge bleibt die Pressekonzentration relativ stabil (vgl. Tabelle 10; sowie WAN-IFRA, 2015). Damit liegt der Marktanteil der grossen Drei in Belgien höher als in der Schweiz sogar fast bei vier Fünftel, ein Sprung von 2011 zu 2012 oder danach ist nicht zu erkennen.

Hinsichtlich Österreichs ist anzumerken, dass es das einzige Untersuchungsland ist, welches im Untersuchungszeitraum hinsichtlich der Gesamtauflage eine leichte Steigerung aufweist (vgl. Tabelle A.4). Unangefochtener Marktführer im Bereich Tageszeitungen ist in Österreich die Mediaprint Zeitungs- und Zeitschriften $\mathrm{GmbH}$ (folgend Mediaprint), welche im Jahr 2009 einen Marktanteil von 34.2 Prozent aufweist und die zweitplatzierte Ultimate Media $\mathrm{GmbH}$, welche einen Marktanteil von 18.5 Prozent aufweist, bereits um 15.7 Prozentpunkte distanziert. An dritter Stelle folgt die Styria Media Group mit einem Marktanteil von 13.2 Prozent. Im Zeitraum 2009 zu 2010 findet kein externes Wachstum, weder positiv noch negativ, hinsichtlich der drei grössten Medienunternehmen statt. Die Auflagen der drei Marktführer bleiben nahezu stabil, was bei gleichzeitig steigender Gesamtauflage Österreichs (1.9 Prozent) in diesem Zeitraum (vgl. Tabelle A.4) zu einer leichten Abnahme der Marktanteile führt. Dies wiederum resultiert in einem abnehmenden CR3-Wert, welcher von 2010 (65.8 Prozent) zu 2011 (65 Prozent) um 0.8 Prozent sinkt (vgl. Tabelle 10; sowie WAN-IFRA, 2015).

Von $2010 \mathrm{zu} 2011$ steigt der CR3-Wert um 3.3 Prozentpunkte auf einen Wert von
68.3 Prozent. Während die Gesamtauflage Österreichs in diesem Zeitraum um 6.5 Prozent steigt (vgl. Tabelle A.4), ist es auch den drei Marktführern der Jahre 2009 und 2010 möglich, ihre Auflagen in ungefähr gleichem Masse zu steigern, so dass ausschliesslich marginale Veränderungen hinsichtlich ihrer Marktanteile entstehen. Der Auslöser für den Anstieg des CR3-Wertes ist die enorme Auflagensteigerung der Gratistageszeitung Österreich, die von der Mediengruppe Österreich GmbH herausgegeben wird. Diese steigert ihre Auflage z.B. von 2010 zu 2011 um 42.7 Prozent und erreicht damit einen Marktanteil von 16.7 Prozent, womit sie die Styria Media Group, welche im Jahr 2011 einen Marktanteil von 13.1 Prozent aufweist, vom dritten Platz verdrängt (vgl. Tabelle 10; sowie WAN-IFRA, 2015).

Zum Jahr 2012 sinkt die Auflage der Gratistageszeitung Österreich um 34.5 Prozent, so dass sich die Styria Media Group erneut auf dem dritten Platz positioniert. Die Gesamtauflage Österreichs sinkt in diesem Zeitraum um 2.9 Prozent (vgl. Tabelle A.4). Im Hinblick auf die drei Marktführer finden abermals keine externen Wachstumsprozesse statt, jedoch sinkt die Gesamtauflage der Mediaprint und der Styria Media Group durch negatives internes Wachstum. Der Marktanteil der Mediaprint sinkt um 0.7 Prozentpunkte auf einen Wert von 32 Prozent. Der Marktanteil der Styria Media Group sinkt um 0.5 Prozentpunkte auf einen Wert von 12.6 Prozent. Ausschliesslich Ultimate Media kann seine Auflage entgegen dem Trend ausbauen und steigert damit den Marktanteil um 1.0 Prozentpunkte auf einen Wert von 19.8 Prozent. Durch den starken Auflagenrückgang der Gratistageszeitung Österreich und die leichten Veränderungen hinsichtlich Mediaprints sowie der Styria Media Group nimmt der CR3-Wert von 2011 zu 20123.9 Prozentpunkte auf einen Wert von 64.4 Prozent ab. In 2013 und 2014 erreicht der Konzentrationswert aber ebenfalls die Regionen von der Schweiz und Belgien weil es im Jahr 2013 einen neuen Auflagensprung bei der Österreich um 42.9 Prozent gibt (vgl. Tabelle 10; sowie WAN-IFRA, 2015). 
Irland weist im Jahr 2009 einen CR3Wert von 61.8 Prozent auf. Marktführer in diesem Jahr ist mit einem Marktanteil von 37.4 Prozent Independent News \& Media, gefolgt von der Irish Times Ltd. mit 14.3 Prozent, sowie News Corp mit 10.1 Prozent Marktanteil. Im Zeitraum von $2009 \mathrm{zu} 2010$ ist zuvorderst der starke Auflagenrückgang um 16.4 Prozent zu bemerken (vgl. Tabelle A.4). Weiterhin erfolgt zum Januar 2010 die Fusion der zwei Gratistageszeitungen Herald $A M$ und Metro zu einem Titel, welcher bis dato unter dem Namen Metro Herald geführt wird (vgl. Metro Herald, 2014). Der Herald $A M$ gehörte Independent News \& Media, während die Metro ein Joint Venture aus DMGT plc., der Irish Times Ltd. sowie Metro International war, welche jeweils ein Drittel der Anteile hielten (vgl. Metro International, 2005). Hinsichtlich des Metro Herald bekam Independent News \& Media die Möglichkeit, die Anteile von Metro International zu übernehmen, so dass der Metro Herald bis 2014 ein Joint Venture - anteilig zu jeweils einem Drittel - aus Independent News \& Media, der Irish Times Ltd. sowie der DMGT plc. war (vgl. Metro Herald, 2014; WAN-IFRA, 2010, S. 265). Bedingt durch den kompletten Auflagenverlust der Herald AM (negatives externes Wachstum) sowie Auflagenrückgang bei den bezahlten Tageszeitungen The Irish Independent und dem Evening Herald (negatives internes Wachstum), welche nicht durch die Anteile an der Auflage des Metro Herald (positives externes Wachstum) ausgeglichen werden können, verliert Independent News \& Media von 2009 zu 20101.9 Prozentpunkte hinsichtlich ihres Marktanteils. Die zweitplatzierte Irish Times Ltd. verliert im gleichen Zeitraum 6 Prozent an Auflage, da dies in Relation zum Gesamtauflagenverlust Irlands (vgl. Tabelle A.4) eine geringere Abnahme darstellt, steigt der Marktanteil um 1.8 Prozentpunkte auf einen Wert von 16.1 Prozent im Jahre 2010. Die drittplatzierte News Corp verliert von 2009 zu 2010 ebenfalls an Auflage ( -8.7 Prozent), kann äquivalent zur Irish Times Ltd. in Relation zum Gesamtauflagenrückgang Irlands den Marktanteil jedoch um 1.0 Prozentpunkte auf einen Wert von 11.1 Prozent (2010) ausbauen. Die Auswirkungen der beschriebenen Veränderungen auf den CR3Wert sind mit 0.8 Prozentpunkten Anstieg von einem Wert von 61.8 Prozent im Jahr 2009 auf einen Wert von 62.6 Prozent im Jahr 2010 gekennzeichnet (vgl. Tabelle 10; sowie WAN-IFRA, 2015).

Der Zeitraum der Jahre 2010 zu 2011 ist von einem Rückgang der Gesamtauflage Irlands um 9.3 Prozent gekennzeichnet (vgl. Tabelle A.4). Des Weiteren finden in diesem Zeitraum keine Prozesse externen Wachstums statt. Der Marktführer Independent News \& Media sowie die drittplatzierte News Corp weisen ein leicht negatives internes Wachstum von $4.6 \mathrm{bzw}$. 4.8 Prozent auf. Da dieses in Relation zum Auflagenrückgang Irlands im gleichen Zeitraum geringer ausfällt, steigen die Marktanteile zum Jahre 2011 um 1.8 Prozentpunkte auf einen Wert von 37.3 Prozent hinsichtlich Independent News \& Media sowie 0.2 Prozentpunkte auf einen Wert von 11.3 Prozent in Bezug auf News Corp. Die Zweitplatzierte Irish Times Ltd. hält ihre Auflage von 2010 zu 2011 annähernd konstant, womit sie im Verhältnis zur sinkenden Gesamtauflage Irlands ihren Marktanteil rechnerisch um 1.4 Prozentpunkte auf einen Wert von 17.5 Prozent ausbaut. Durch die Steigerung der Marktanteile der drei grössten Medienunternehmen im Bereich Tageszeitungen erhöht sich der CR3-Wert von $2010 \mathrm{zu} 2011$ um 3.4 Prozentpunkte auf einen Wert von 66.1 Prozent im Jahre 2011 (vgl. Tabelle 10; sowie WAN-IFRA, 2015).

Die weitere Entwicklung zum Jahre 2012 ist den vorherigen ähnlich: Der Trend des Rückgangs der Gesamtauflage Irlands (-6.7 Prozent) hält weiter an (vgl. Tabelle A.4). Die drei Marktführer verlieren leicht an Auflage, was sich in geringen Veränderungen der Marktanteile (Independent News \& Media (+0.1 Prozentpunkte), The Irish Times Ltd. (-0.3 Prozentpunkte), News Corp (-0.3 Prozentpunkte)) äussert. Dies wirkt sich ebenfalls mindernd auf den CR3 aus, welcher zum Jahr 2012 um 0.5 Prozentpunkte auf einen Wert von 65.6 und 2013 weiter auf 63.5 sinkt. Im Jahr 2014 gibt es - wenn nur die Zeitungen in Be- 
tracht gezogen werden, die am 31.12.2014 noch existiert haben - aber einen grossen Sprung auf beinahe drei Viertel des Marktes, so dass alle vier Kleinstaaten sehr ähnliche Pressekonzentrationsraten nach der CR-3-Methode aufweisen (vgl. Tabelle 10; sowie WAN-IFRA, 2015). Auch hier fällt es schwer, einen Zusammenhang zur gesteigerten Förderung festzuhalten.

Vom Jahr 2010 zu 2012 sinkt der CR3 Deutschlands leicht um 0.3 Prozentpunkte auf einen Wert von 33.7 und 2014 weiterauf 32.7. Während die marktführende Axel Springer AG in diesem Zeitraum weder Zu- noch Verkäufe tätigt, verliert sie vor allem durch den aus den Vorjahren anhaltenden deutlichen Auflagenrückgang der Bild-Zeitung an Marktanteil. Dieses negative interne Wachstum verdeutlicht sich am Wert des Marktanteils von 18.8 Prozent im Jahr 2012, was einem Verlust von 0.8 Prozentpunkten im Vergleich zu 2010 darstellt. Die Verlagsgruppe Süddeutsche Zeitung/Die Rheinpfalz/ Südwest Presse hat hinsichtlich externen Wachstums im Zeitraum von $2010 \mathrm{zu} 2012$ den Oranienburger Generalanzeiger der Ippen-Gruppe übernommen. Weiterhin konnte sie durch internes Wachstum den Marktanteil vergrössern und steigert ihren Wert von 2010 auf 2012 um 0.6 Prozentpunkte auf einen Wert von 9.2 Prozent. Die WAZ-Gruppe verbleibt in diesem Zeitraum unverändert und verliert marginal an Marktanteil durch leicht negatives internes Wachstum von 0.1 Prozentpunkten, was einen Wert hinsichtlich des Jahres 2012 von 5.7 Prozent ausmacht (vgl. Röper, 2012, S. 272-275; sowie Tabelle 10 und WAN-IFRA, 2015).

Bei zusätzlicher Berücksichtigung des deutschen CR3-Wertes von 36.6 im Jahr 2008 (vgl. Röper, 2010, S. 222) ergibt sich in der Summe durch die sinkende Auflage der Axel Springer AG einerseits und die steigenden Auflagen der Verlagsgruppe Süddeutsche Zeitung/Die Rheinpfalz/ Südwest Presse sowie der WAZ-Gruppe anderseits ein leicht rückläufiger CR3Wert. Zuvorderst ist dies jedoch durch den deutlichen Rückgang der Auflage der Bild-Zeitung im Untersuchungszeitraum begründet (vgl. Tabelle 10; sowie WAN-IFRA, 2015).

\section{Diskussion und Fazit}

Die hier vorgestellte Analyse der Auswirkung von Presseförderung auf die Medienkonzentration im Tageszeitungsmarkt hat zunächst keinen einfachen systematischen Zusammenhang zwischen der Höhe der Subventionierung und der Marktmacht der drei grössten Tageszeitungsverleger ergeben:

1. Es ist nicht so, dass diejenigen kleinen Länder die höchste Medienkonzentration aufweisen, die die Medien am wenigsten finanziell unterstützen, wie sich u.a. an den österreichischen Daten zeigt, auch wenn der Vergleich der absoluten Geldmengen nicht unbedingt auf validen Füssen stehen muss (vgl. Kapitel 5, besonders die Fussnoten 17 und 18).

2. Hinzu kommt, dass steigende Fördersummen in dieser kurz- bis mittelfristigen Analyse nicht zu sinkender oder stagnierender Pressekonzentration führen: In der Schweiz kann z.B. die Aufstockung der Subventionierung kleiner Zeitungen durch günstige Postgebühren nicht verhindern, dass der Marktführer Tamedia weiter wächst, zumal er offensichtlich auch der grösste Profiteur der Subventionierung ist (vgl. Schwab, 2016). In Zukunft wird zu untersuchen sein, ob die eingesetzten Geldmengen gleichgewichtet miteinander verglichen werden sollten, wie das Beispiel aus Fussnote 21 zur Anhebung der Transportgebühren durch die Schweizer Post zeigt. Grundsätzlich Ähnliches gilt für Irland, wo trotz steigender Subventionen 2014 die höchste Pressekonzentration im Untersuchungszeitraum gemessen wurde.

3. Auch bei kontinuierlich sinkender Subventionierung wie in Österreich, steigt die Pressekonzentration nicht kontinuierlich im Jahresabstand. Allerdings ergeben sich 2013 und 2014 sehr hohe Konzentrationswerte, so dass ein län- 
gerfristiger Zusammenhang nicht auszuschliessen ist. In Deutschland sinkt der Subventionierungsbetrag durch den Rückgang der Auflagen und Erträge ebenfalls stetig und die Pressekonzentration des CR-3 Wertes sinkt mit, obwohl Röper (vgl. 2014) von den höchsten CR-10 Werten spricht.

4. Ein systematischer Zusammenhang zwischen der Vielfalt der eingesetzten Fördermassnahmen und der Höhe der Aufwendungen auf der einen Seite und der Medienkonzentration auf der anderen lässt sich in kleinen Ländern nicht festmachen. Systematische Unterschiede zwischen den Mediensystemtypen sind ebenfalls nicht zu entdecken.

Wenn die österreichische Besonderheit der Anzeigenschaltungen ausser Acht gelassen wird, zeigt sich tendenziell, dass es nicht ausreicht, ausschliesslich auf Mehrwertsteuer-Reduktion zu setzen. So senkt bspw. Irland sogar die verminderte Mehrwertsteuer, jedoch steigen die Konzentrationsraten an. $\mathrm{Ob}$ also direkte selektive Presseförderung wirtschaftlich effizienter auf schwache Zeitungen wirkt und somit produktive Effizienz gesteigert werden kann, müssen weitere Studien zeigen, die nicht nur Österreich und Belgien als diversifiziert die Presse fördernde Länder untersuchen. In Belgien ist zwar die Gesamtsumme der Förderung gesunken, die direkte Förderung aber gestiegen. Trotzdem ist die Pressekonzentration hier nahezu stabil mit leicht steigender Tendenz. Weitergehende Studien sollten bspw. Frankreich einbinden, welches erstens vielfältige Presseförderungsmassnahmen etabliert hat und diese darüber hinaus im Jahre $2009 \mathrm{im}$ Zuge des Sarkozy-Plans einen signifikanten Ausbau erfuhren.

Insgesamt erscheint es lohnend, solche Untersuchungen weiterzuführen und im Quer- (alle EU-Länder sowie Schweiz und Norwegen) und Längsschnitt auszudehnen. Allerdings ist kritisch anzumerken, dass die interne Validität bei diesem quasi-experimentellen Versuchsaufbau eher auf tönernen Füssen steht, da Drittvariablen schwierig $\mathrm{zu}$ kontrollieren sind und eine Isolation des zu erforschenden Wirkzusammenhangs nahezu unmöglich ist. Klar ist auch, dass die Setzung der abhängigen Variable auf die drei grössten Medienunternehmen zwar methodisch begründet erfolgt ist, aber keine inhaltliche Verbindung zum Untersuchungsgegenstand besteht: Es ist nicht besonders gefährlich für die Pressevielfalt, wenn drei Unternehmen grosse Meinungsmacht haben. Es können auch fünf, sieben oder zehn sein. Für einen Vergleich verschiedener Koeffizienten, wie Kolb (vgl. 2015) ihn für die Vielfaltsbestimmung im Fernsehbereich durchführt, reichen die vorliegenden Daten nicht aus. Die kurzfristige Alternative jedoch wäre es, nicht $\mathrm{zu}$ forschen, was auch keine - in jedem Fall nicht die beste - Lösung darstellt. Die Autoren arbeiten aktuell an einer Verbesserung der Datenlage (vgl. Kolb \& Oertel, 2016).

$\mathrm{Ob}$ sich die Ergebnisse auf andere Kleinstaaten übertragen lassen, kann nicht bestimmt werden. Im Vergleich $\mathrm{zu}$ dem einzigen Grossstaat Deutschland wird deutlich, dass die Marktgrösse offenbar einen starken Einfluss auf die Medienkonzentration hat: Der CR3-Wert von Deutschland ist deutlich niedriger als bei allen untersuchten Kleinstaaten. Für ein abschliessendes Urteil bezüglich des Einflusses von Marktgrösse und Mediensystemtypus wird jedoch eine Ausweitung der Stichproben auf mehr Länder, ggf. andere Konzentrationswerte (vgl. Röper, 2014) und längerfristige Zeitreihen benötigt. Die Bedeutung solcher Untersuchungen wird besonders deutlich, weil sich eine steigende Medienkonzentration mit dieser Untersuchung bestätigen lässt. Allenfalls in Deutschland steigen die CR3-Werte im Untersuchungszeitraum nicht an sondern stagnieren oder gehen leicht zurück. Ob eine solche Stagnation - bei gleichzeitigem Anstieg in einem anderen Koeffizienten (vgl. Röper, 2014) - im Lichte der demokratietheoretischen Probleme bereits einen ausreichenden Erfolg darstellt, ist (zumindest) fraglich und sollte auf Basis validierter Zahlen für mehr Länder im Zentrum weiterer komparativer Forschung stehen. 


\section{Literaturverzeichnis}

Aarebrot, F.H., \& Bakka, P. H. (2003). Die vergleichende Methode in der Politikwissenschaft. In D. Berg-Schlosser, \& F. Müller-Rommel (Hrsg.), Vergleichende Politikwissenschaft (4., überarbeitete und erweiterte Auflage, S. 57-76). Opladen: Leske + Budrich

Altmeppen, K.-D. (2013). Medienkonzentration. In G. Bentele, H.-B. Brosius, \& O. Jarren (Hrsg.), Lexikon Kommunikations- und Medienwissenschaft (S. 211-213). Wiesbaden: Springer VS.

Aslama, M., De Bens, E., van Cuilenburg, J., Nordenstreng, K., Schulz, W., \& van der Wurff, R. (2007). Measuring and Assessing Empirical Media Diversity: Some European Cases. In E. de Bens (Hrsg.), Media Between Culture and Commerce. Changing Media - Changing Europe Series Vol. 4 (S. 55-98). Bristol, Chicago: intellect.

Atteslander, P. (2010). Methoden der empirischen Sozialforschung (ESV basics, 13., neu bearb. und erw. Aufl). Berlin: Erich Schmidt.

Axel Springer AG (2013). Gemeinsame Presseinformation der FUNKE MEDIENGRUPPE und der Axel Springer $A G$, Axel Springer AG. Zugriff am 10.03.2014. Verfügbar unter http://www.axelspringer.de/ presse/FUNKE-MEDIENGRUPPEuebernimmt-Regionalzeitungen-undTeile-des-Zeitschriftenportfoliosvon-Axel-Springer-Gruendungvon-Gemeinschaftsunternehmenfuer-Anzeigenvermarktung-undVertrieb_18994020.html

Baker, C. E. (2007). Media Concentration and Democracy. Why Ownership Matters (Communication, society, and politics). Cambridge: Cambridge University Press.

BDZV (2007). BDZV: Zur Lage der Zeitungen in Deutschland 2007, BDZV. Zugriff am 23.08.2014. Verfügbar unter http://www. bdzv.de/markttrends-und-daten/wirtschaftliche-lage/artikel/detail/zur_lage_ der_zeitungen_in_deutschland_2007/

Beck, K. (2013). Funktion. In G. Bentele, H.-B. Brosius, \& O. Jarren (Hrsg.), Lexikon Kommunikations- und Medienwissenschaft (S. 98-99). Wiesbaden: Springer VS.
Bonfadelli, H., \& Schwarb, U. (2006). Medienkonzentration und publizistische Vielfalt. In H. Bonfadelli, W.A. Meier, \& J. Trappel (Hrsg.), Medienkonzentration Schweiz: Formen, Folgen, Regulierung. (S. 21-40). Haupt.

Blum, R. (2014). Lautsprecher und Widersprecher. Ein Ansatz zum Vergleich der Mediensysteme. Köln: Herbert von Halem.

Bortz, J., \& Döring, N. (2006). Forschungsmethoden und Evaluation. Für Human- und Sozialwissenschaftler (Springer-Lehrbuch, 4., überarbeitete Aufl). Heidelberg: Springer Medizin.

Brüggemann, M., Engesser, S., Büchel, F., Humprecht, E., \& Castro, L. (2014). Hallin and Mancini Revisited: Four Empirical Types of Western Media Systems. Journal of Communication, 64(6), 1037-65.

Bundesamt für Statistik. (2015). Sprachen, Religionen - Daten, Indikatoren, Bundesamt für Statistik. Zugriff am 21.07.2015.

Central Statistics Office. (2012). Census 2011: Profile 9. What We Know, Central Statistics Office. Zugriff am 13.08.2014. Verfügbar unter http://www.cso.ie/en/media/csoie/ census/documents/census2011profile9/ Profile,9,What,we,know,full,doc,for,web. pdf

Centre d'Information sur les Médias (2015). Tableaux récapitulatifs 2014-T1 / 2014-T4. Zugriff am 23.10.2016. Verfügbar unter www.cim.be/fr/cim/download/press_authentication_reports?file=resume_table/2014q4ResumeTable_fr.pdf

Debouté, A., \& Renault, E. (2014, 12. März). Bolloré et TF1 étudient le rachat de 20 Minutes. Le Figaro. Zugriff am 03.09.2014. Verfügbar unter http:/ /www.lefigaro.fr/ medias/2014/03/12/20004-20140312ARTFIG00021-bollore-et-tf1-etudient-lerachat-de-20-minutes.php

Dreier, H. (2006). Konzentration. In HansBredow-Institut (Hrsg.), Medien von A biz Z (S. 185-188). Wiesbaden: VS Verlag für Sozialwissenschaften.

Europäische Zentralbank. (2014). ECB: Euro Foreign Exchange Reference Rates, Europäische Zentralbank. Zugriff am 18.07.2014. Verfügbar unter https://www.ecb.europa. $\mathrm{eu} /$ stats/exchange/eurofxref/

Freedman, D. (2008). The Politics of Media Policy. Cambridge: Polity Press. 
Frühschütz, J. (2004). Horizont Medien-Lexikon. Frankfurt am Main: Deutscher Fachverlag.

Groupe Rossel. (2015). Le Groupe Rossel se rapproche de "20 Minutes». Zugriff am 16.10.2016. Verfügbar unter http://www. lefigaro.fr/medias/2014/03/12/2000420140312ARTFIG00021-bollore-et-tf1etudient-le-rachat-de-20-minutes.php

Haas, H. (2012). Evaluierung der Presseförderung in Österreich. Status, Bewertung, internationaler Vergleich und Innovationspotenziale. Eine Studie im Auftrag des Bundeskanzleramtes Österreich. Wien: Universität Wien: Universität Wien. Zugriff am 25.08.2014. Verfügbar unter https:// www.bka.gv.at/DocView.axd?CobId=50443

Hallin, D. C., \& Mancini, P. (2004). Comparing Media Systems. Three Models of Media and Politics. Cambridge: Cambridge University Press.

Heinrich, J. (2010). Medienökonomie. Band 1: Mediensystem, Zeitung, Zeitschrift, Anzeigenblatt (Medienökonomie, Bd. 1, 3. Aufl). Wiesbaden: VS Verlag für Sozialwissenschaften.

Holtz-Bacha, C. (1994). Presseförderung im westeuropäischen Vergleich. In P.A. Bruck (Hrsg.), Medienmanager Staat. Von den Versuchen des Staates, Medienvielfalt zu ermöglichen (Ökonomie und Zukunft der Printmedien, Bd. 2, S. 443-567). Medienpolitik im internationalen Vergleich. München: R. Fischer.

Hugentobler, A., \& Jaag, C. (2013). Switzerland: The Role of Swiss Post in Indirect Press Support. In P. Murschetz (Hrsg.), State Aid for Newspapers (Media Business and Innovation, S. 323-336). Berlin: Springer.

Imhof, K., Blum, R., Bonfadelli, H., \& Jarren, O. (2006). Einleitung. In K. Imhof, R. Blum, H. Bonfadelli, \& O. Jarren (Hrsg.), Demokratie in der Mediengesellschaft (S. 9-24). Wiesbaden: VS Verlag für Sozialwissenschaften.

Karstens, E., \& Schütte, J. (2010). Praxishandbuch Fernsehen. Wie TV-Sender arbeiten. Wiesbaden: VS Verlag für Sozialwissenschaften.

Kleinsteuber, H. J. (2003). Medien und Kommunikation im internationalen Vergleich: Konzepte, Methoden und Befunde. In F. Esser, \& B. Pfetsch (Hrsg.), Politische Kom- munikation im internationalen Vergleich. Grundlagen, Anwendungen, Perspektiven (S. 78-103). Wiesbaden: Westdeutscher Verlag.

Knoche, M. (2007). Medienkonzentration. In B. Thomass (Hrsg.), Mediensysteme im internationalen Vergleich (S. 122-144). Konstanz: UVK.

Knoche, M. (1999). Medienkonzentration und publizistische Vielfalt. Legitimationsgrenzen des privatwirtschaftlichen Mediensystems. In R. Renger, \& G. Siegert (Hrsg.), Kommunikationswelten. Wissenschaftliche Perspektiven zur Medien- und Informationsgesellschaft (Beiträge zur Medien- und Kommunikationsgesellschaft, Bd. 1, 2., korrigierte Auflage, S. 123-158). Innsbruck: Studien Verlag.

Kolb, S. (2015). Vielfalt im Fernsehen. Eine komparative Studie zur Entwicklung von TV-Märkten in Westeuropa (Forschungsfeld Kommunikation, Bd. 35). Konstanz: UVK.

Kolb, S., \& Beck, D. (2011). Vergleichbarkeit in der (international) vergleichenden Journalismusforschung auf der Basis von Sekundäranalysen. In O. Jandura; T. Quandt, \& J. Vogelgesang (Hrsg.), Methoden der Journalismusforschung (S. 351-365). Wiesbaden: VS Verlag für Sozialwissenschaften.

Kolb, S., \& Lüdi, M. (2016). Wettbewerb im regionalen Fernsehen mit Gebührensubvention? In A. Fehr, S. Fiechtner, \& J. Trebbe, (Hrsg.): Über Grenzen hinweg. Fernsehberichterstattung in der Schweiz (im Druck). Berlin, LIT.

Kolb, S., \& Oertel, M. (2016). Quasi-experimentelle Modellierung in (international) vergleichenden Sekundäranalysen zur Erforschung von Medienstrukturen am Beispiel der Presseförderung in Europa. In B. von Rimscha; S. Studer, \& M. Puppis (Hrsg.), Methodische Zugänge zur Erforschung von Medienstrukturen, Medienorganisationen und Medienstrategien (S. 136-153). Baden-Baden: Nomos.

Künzler, M., Puppis, M., Schweizer, C., \& Studer, S. (2013): Monitoring-Report «Medienförderung». RegulierungsMonitoring 2012/2013. IPMZ - Institut für Publizistikwissenschaft und Medienforschung, Universität Zürich. 
Kust, H., \& Kolb, S. (2013). Kurzprofile der Regionalsender. In S. Kolb, G. Baeva, \& B. Schwotzer (Hrsg.), Gebühren gleich Qualität? Inhaltsanalyse der Schweizer Regionalfernsehprogramme (S. 21-45). Chur: Rüegger.

Marcourt, J.-C. (2016). Aides à la presse écrite. Zugriff am 21.10.2016. Verfügbar unter http://marcourt.wallonie.be/aides-a-lapresse-ecrite/

McDonald, D. G., \& Dimmick, J. (2003). The Conceptualization and Measurement of Diversity. Communication Research, 30(1), 60-79.

McQuail, D. (1992). Media Performance. Mass Communication and the Public Interest. London: Sage.

Metro Herald. (2014). Metro Herald. Zugriff am 11.03.2014. Verfügbar unter http://www. metroherald.ie/howitallstarted.html

Metro International. (2005). Partnership to launch in Ireland, Metro International. Zugriff am 01.09.2014. Verfügbar unter http://www.metro.lu/lang/en/press_ release/partnership-to-launch-metro-inireland/

Molloy, T. (2014). Metro Herald to cease publication on Friday. Zugriff am 23.10.2016. Verfügbar unter http:/ / www.independent. ie/business/media/metro-herald-to-cease-publication-on-friday-30839023.html

Murschetz, P. (1998). State Support for the Daily Press in Europe: A Critical Appraisal: Austria, France, Norway and Sweden Compared. European Journal of Communication, 13(3), 291-313.

Murschetz, P. (2013a). The Context, Purpose, and Structure of the Book. In P. Murschetz (Hrsg.), State Aid for Newspapers (Media Business and Innovation, S. 3-19). Berlin: Springer.

Murschetz, P. (2013b). State Aid for Newspapers: First Theroetical Disputes. In P. Murschetz (Hrsg.), State Aid for Newspapers (Media Business and Innovation, S. 21-46). Berlin: Springer.

Murschetz, P. (2013c). State Aid for Newspapers (Media Business and Innovation). Berlin: Springer.

Murschetz, P., \& Karmasin, M. (2013). Austria: Press Subsidies in Search of a New Design. In P. Murschetz (Hrsg.), State Aid for News- papers (Media Business and Innovation, S. 133-148). Berlin: Springer.

Napoli, P. M. (1999). Deconstructing the Diversity Principle. Journal of Communication, 49(4), 7-34.

Napoli, P. M. (2001). Foundations of Communication Policy. Principles and Process in the Regulation of electronic Media (The Hampton Press communication series). Cresskill: Hampton.

Niedermayer, O., \&Widmaier, U. (2003). Quantitativ vergleichende Methoden. In D. Berg-Schlosser, \& F. Müller-Rommel (Hrsg.), Vergleichende Politikwissenschaft (4., überarbeitete und erweiterte Auflage, S. 77-101). Opladen: Leske + Budrich.

NZZ Mediengruppe (Hrsg.). (2010). FPH Freie Presse Holding übernimmt Thurgauer Zeitung und verkauft Tamedia ihre Zürcher Landzeitungs-BeteiligungenVerlegerfamilie Gut verkauft Mehrheitsbeteiligung an Zürichsee Zeitung an Tamedia. Zugriff am 20.07.2015. Verfügbar unter http://www.nzzmediengruppe. ch/2010/04/15/fph-freie-presse-holding-uebernimmt-thurgauer-zeitung-und-verkauft-tamedia-ihre-zuercher-landzeitungs-beteiligungen-verlegerfamilie-gut-verkauft-mehrheitsbeteiligung-an-zuerichsee-zeitung-an-tamedia/ Oertel, M. (2014). Der Einfluss von Presseförderung auf Medienkonzentration und publizistische Vielfalt im Bereich Tageszeitungen. Eine komparative Längsschnitt-sowie Querschnittsuntersuchung von sieben Staaten der Europäischen Union. Masterarbeit. Berlin.

Parliament of the German Community (2015). Population Density, Deutschsprachige Gemeinschaft Belgiens. Verfügbar unter http://www.dglive.be/en/desktopdefault. aspx/tabid-1407/2266_read-27183/

Pelinka, A. (2005). Vergleich politischer Systeme (UTB. Politikwissenschaft, Bd. 2726). Wien: Böhlau.

Picard, R. G. (2006). Issues and challenges in the provision of press subsidies. In I. Fernandez Alonso; M. de Moragas, J. J. Blasco Gil, \& N. Almiron (Hrsg.), Press subsidies in Europe (S. 211-220). Barcelona: Generalitat de Catalunya. Zugriff am 16.10.2016. Verfügbar unter http://www.portalcomu- 
nicacion.com/opc/download/press_eng. pdf

Picard, R. G. (2002). The Economics and Financing of Media Companies. New York: Fordham University Press.

Przeworski, A., \& Teune, H. (1970). The Logic of Comparative Social Inquiry (Comparative studies in behavioral science). New York: Wiley-Interscience.

Puppis, M. (2010). Einführung in die Medienpolitik (UTB, 2881 : Medien- und Kommunikationswissenschaft, 2., überarb. Aufl). Stuttgart: UVK.

Puppis, M. (2009). Media Regulation in Small States. International Communication Gazette, 71(1-2), 7-17.

Puppis, M., Künzler, M., \& Jarren, O. (Hrsg.). (2013). Media Structures and Media Performance. Medienstrukturen und Medienperformanz (Relation, n.s., Vol. 4). Wien: Verlag der Österreichischen Akademie der Wissenschaften (ÖAW).

Rager, G., \&Weber, B. (Hrsg.). (1992). Publizistische Vielfalt zwischen Markt und Politik. Mehr Medien - mehr Inhalte? Düsseldorf: Econ.

Regierung der Deutschsprachigen Gemeinschaft Belgiens. (2016). Erlass der Regierung zur Gewährung von Subventionen an die geschriebene Presse der Deutschsprachigen Gemeinschaft (174000.00 EURO). Zugriff am 21.10.2016. Verfügbar unter http://www.dgregierung.be/desktopdefault.aspx/tabid-2988/620_read-47218/

Röper, H. (2014). Zeitungsmarkt 2014: Erneut Höchstwert bei Presskonzentration. Media Perspektiven, 45(5), 254-270. Zugriff am 25.07.2016. Verfügbar unter http://www. ard-werbung.de/fileadmin/user_upload/ media-perspektiven/pdf/2014/05-2014_ Roeper.pdf

Röper, H. (2012). Zeitungsmarkt 2012: Konzentration erreicht Höchstwert. Daten zur Konzentration der Tagespresse in der Bundesrepublik Deutschland im I. Quartal 2012. Media Perspektiven, 43 (5), 268-285. Zugriff am 19.06.2014. Verfügbar unter http://www.media-perspektiven.de/ uploads/tx_mppublications/05-2012_Roeper_01.pdf

Röper, H. (2010). Zeitungen 2010: Rangverschiebungen unter den grössten Verlagen. Daten zur Konzentration der Tages- presse in der Bundesrepublik Deutschland im I. Quartal 2010. Media Perspektiven, 41(5), 218-234.

Sarcinelli, U. (1998). Politikvermittlung und Demokratie: Zum Wandel der politischen Kommunikationskultur. In U. Sarcinelli (Hrsg.), Politikvermittlung und Demokratie in der Mediengesellschaft. Beiträge zur politischen Kommunikationskultur (S. 11-23). Opladen: Westdeutscher Verlag. Schnell, R., Esser, E., \& Hill, P. B. (2013). Methoden der empirischen Sozialforschung (10., überarb. Aufl). München: Oldenbourg.

Schütz, W.J. (2012). Deutsche Tagespresse 2012. Ergebnisse der aktuellen Stichtagssammlung. Media 43(11), 594-603. Zugriff am 16.10.2016. Verfügbarunter http://www. ard-werbung.de/fileadmin/user_upload/ media-perspektiven/pdf/2012/11-2012_ Schuetz_2.pdf

Schwab, I. (2016). Wer profitiert von der indirekten Presseförderung? Erstmals Zahlen zur umstrittenen Mediensubvention. Zugriff am 22.10.2016. Verfügbar unter: http://medienwoche.ch/2016/10/13/ wer-profitiert-von-der-indirekten-pressefoerderung/

Schwarb, U. (2007). Medienvielfalt und publizistische Leistung. 20 Jahre nach Einführung des lokalen Rundfunks in der Schweiz. Konstanz: UVK.

Statistik Austria. (2013). Presseförderung des Bundes 2009 bis 2012, Statistik Austria. Zugriff am 14.03.2014. Verfügbar unter http://www.statistik.at/web_de/statistiken/bildung_und_kultur/kultur/buecher_ und_presse/029410.html

Tamedia AG. (2014). Tamedia AG - GeschichteNachrichten, Medien, Verlag, Zeitung, Zeitschrift, Elektronische Medien, Tamedia AG. Zugriff am 05.03.2014. Verfügbar unter http://www.tamedia.ch/de/unternehmen/tamedia-publications-romandes/ geschichte/

Trappel, J., \& Zettl, V. (2013, 3. Februar). Öffentliche Werbung: Politische Sterntaler. DerStandard.at. Zugriff am 25.08.2014. Verfügbar unter http://derstandard. at/1358305492833/Oeffentliche-Werbung-Politische-Sterntaler

Trebbe, J., Baeva, G., Schwotzer, B., Kolb, S., \& Kust, H (2008): Fernsehprogrammanalyse 
Schweiz Methode, Durchführung, Ergebnisse. Zürich: Rüegger.

Van Cuilenburg, J. (2007). Media Diversity, Competition and Concentration: Concepts and Theories. In E. de Bens (Hrsg.), Media Between Culture and Commerce. Changing Media - Changing Europe Series Vol. 4 (S. 25-54). Bristol, Chicago: intellect. Van Cuilenburg, J., \& van der Wurff, R. (2007). Towards Easy-to-Measure Media Diversity Indicators. In E. de Bens (Hrsg.), Media Between Culture and Commerce. Changing Media - Changing Europe Series Vol. 4 (S. 99-113). Bristol, Chicago: intellect. Verband Schweizer Medien (2014). Die 15 grössten Tageszeitungen 2014. Zugriff am 23.10.2016. Verfügbar unter http:// www.schweizermedien.ch/SCHM/media/ SCHMMediaLibrary/Statistiken/Top15_ Tageszeitungen_Aufl_Leser_04_2014.pdf WAN-IFRA. (2010). World Press Trends Europe 2010, Darmstadt.

WAN-IFRA. (2015). WPT Database - WAN-IFRA World Press Trends Database, WAN-IFRA. Zugriff am 26.06.2015. Verfügbar unter http://www.wptdatabase.org/

Ward, D. (2004). A Mapping Study of Media Concentration and Ownership in Ten European Countries (Commissariaat voor de Media, Hrsg.), Hilversum. Zugriff am 10.08.2014. Verfügbar unter http://77.87.161.246/wp-content/ uploads/2013/08/A-Mapping-Study-of-Media-Concentration-and-Ownership-in-Ten-European-Countries.pdf

WEMF (2014). WEMF AUFLAGEBULLETIN 2014. Zugriff am 23.10.2016. Verfügbar unter http://www.wemf.ch/pdf/de/auflagen/2014/Bulletin_20141101_d.pdf

Wirth, W., \& Kolb, S. (2003). Äquivalenz als Problem: Forschungsstrategien und Designs der komparativen Kommunikationswissenschaft. In F. Esser, \& B. Pfetsch (Hrsg.), Politische Kommunikation im internationalen Vergleich. Grundlagen, Anwendungen, Perspektiven (S. 104-131). Wiesbaden: Westdeutscher Verlag.

Zerback, T. (2013). Publizistische Vielfalt. Demokratischer Nutzen und Einflussfaktoren. Konstanz: UVK. 


\section{Anhang}

Tabelle A.1: Schätzung der Umsätze bezahlter Tageszeitungen in Österreich für die Jahre 2006-2010, 2013 und 2014

\begin{tabular}{|c|c|c|c|c|c|c|c|c|c|}
\hline & 2006 & 2007 & 2008 & 2009 & 2010 & 2011 & 2012 & 2013 & 2014 \\
\hline $\begin{array}{l}\text { Auflage (in Tausend) } \\
\text { (gesamt bezahlte Tageszeitungen) }\end{array}$ & 2356 & 2406 & 2340 & 2305 & 2009 & 1997 & 1980 & 1862 & 1838 \\
\hline Umsatz effektiv (in tausend EUR) ${ }^{1}$ & - & - & - & - & - & 151984 & 152982 & - & - \\
\hline Verhältniskoeffizient ${ }^{2}$ & - & - & - & - & - & 76.11 & 77.26 & - & - \\
\hline Umsatz geschätzt (in tausend EUR) & 179306 & 183111 & 178088 & 175425 & 152897 & - & - & 143865 & 142011 \\
\hline
\end{tabular}

1 WAN-IFRA (2015).

2 Der Verhältniskoeffizient wiedergibt das Verhältnis zwischen Umsatz und Auflage. Er errechnet sich mittels Division des Umsatz durch die Auflage.

3 Die Umsätze für die Jahre 2006 bis 2010 werden wie folgt geschätzt: Multiplikation der jeweiligen Auflage mit dem Verhältniskoeffizienten 2011. Die Schätzung der Umsätze der Jahre 2013 und 2014 erfolgt mittels Multiplikation der jeweiligen Auflage mit dem Verhältniskoeffizienten 2012. Für die Schätzung der Umsätze wurden die exakten Auflagen verwendet (nicht die Auflage in Tausend)..

Tabelle A.2: $\quad$ Umrechnungskurs CHF in EUR 2009 bis 2014

\begin{tabular}{rrrrrrr}
\hline & 2009 & 2010 & 2011 & 2012 & 2013 & 2014 \\
\hline EUR & 0.674 & 0.798 & 0.822 & 0.828 & 0.814 & 0.832
\end{tabular}

Quelle: eigene Darstellung: 2006-2014 auf Basis der Europäische Zentralbank (2016).

Anmerkung: Der Umrechnungskurs gibt an, welchen Wert ein Schweizer Franken (CHF) am 31.12. des jeweiligen Jahres in Euro (EUR) darstellte.

Tabelle A.3: $\quad$ Bruttoinlandsprodukt 2009 bis 2014 (in Millionen EUR)

\begin{tabular}{lrrrrrr}
\hline Land & 2009 & 2010 & 2011 & 2012 & 2013 & 2014 \\
\hline Schweiz & 535650 & 550571 & 586784 & 592808 & 515680 & 528780 \\
Belgien & 340788 & 354688 & 369981 & 376229 & 392675 & 400408 \\
Österreich & 274818 & 286197 & 300712 & 310847 & 322539 & 330417 \\
Irland & 160597 & 155992 & 158992 & 163595 & 180209 & 193159 \\
Deutschland & 2374500 & 2476800 & 2592600 & 2644200 & 2820820 & 2915650 \\
\hline
\end{tabular}

Quelle: eigene Darstellung auf Basis der WAN-IFRA (2015) sowie Eurostat (2016).

Tabelle A.4: Durchschnittliche Auflage von bezahlten Tageszeitungen und Gratistageszeitungen pro Ausgabe 2009 bis 2014 (Anzahl)

\begin{tabular}{lrrrrrr}
\hline Land & 2009 & 2010 & 2011 & 2012 & 2013 & 2014 \\
\hline Schweiz & 3449000 & 3097000 & 3051000 & 2978000 & 2848700 & 2790000 \\
Belgien & 1634000 & 1622000 & 1598000 & 1564000 & 1520000 & 1496000 \\
Österreich & 2869000 & 2924000 & 3115000 & 3024000 & 2986000 & 2905000 \\
Irland & 909000 & 760000 & 689320 & 643077 & 599690 & 490100 \\
Deutschland & 19746000 & 19090000 & 18522000 & 18021000 & 17241500 & 16306650 \\
\hline
\end{tabular}

Quelle: eigene Darstellung auf Basis der WAN-IFRA (2016). 